\title{
The JG $\beta$-relaxation in water and impact on the dynamics of aqueous mixtures and hydrated biomolecules
}

Cite as: J. Chem. Phys. 151, 034504 (2019); https://doi.org/10.1063/1.5100835

Submitted: 21 April 2019 . Accepted: 19 June 2019 . Published Online: 17 July 2019

S. Capaccioli (D), K. L. Ngai, S. Ancherbak, M. Bertoldo, G. Ciampalini, M. Shahin Thayyil, and Li-Min Wang (i)

\section{COLLECTIONS}

Paper published as part of the special topic on Chemical Physics of Supercooled Water Note: This paper is part of a JCP Special Topic on Chemical Physics of Supercooled Water.
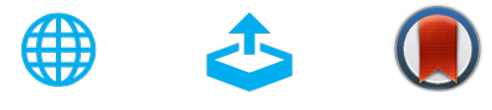

\section{ARTICLES YOU MAY BE INTERESTED IN}

A proposal for the structure of high- and low-density fluctuations in liquid water

The Journal of Chemical Physics 151, 034508 (2019); https://doi.org/10.1063/1.5100875

Water dynamics in the hydration shells of biological and non-biological polymers

The Journal of Chemical Physics 150, 234904 (2019); https://doi.org/10.1063/1.5096392

Is water one liquid or two?

The Journal of Chemical Physics 150, 234503 (2019); https://doi.org/10.1063/1.5096460
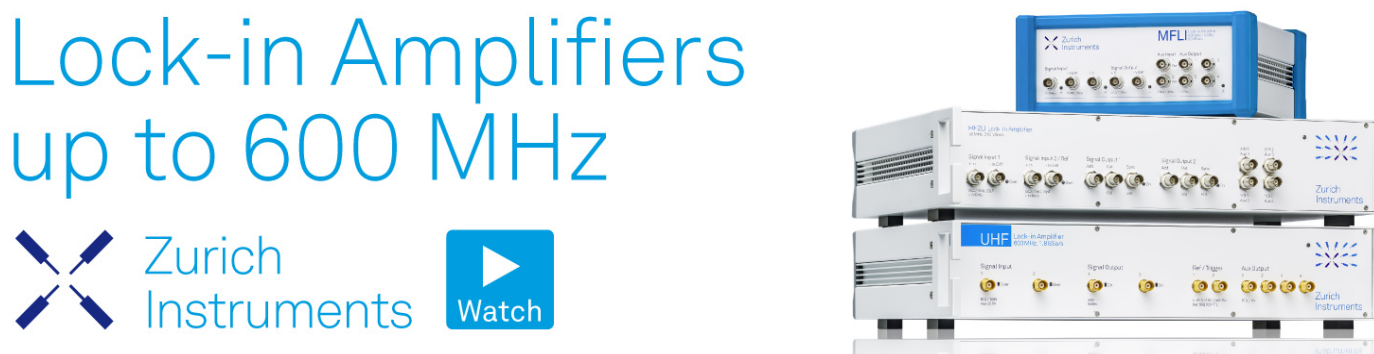


\title{
The JG $\beta$-relaxation in water and impact on the dynamics of aqueous mixtures and hydrated biomolecules
}

\author{
Cite as: J. Chem. Phys. 151, 034504 (2019); doi: 10.1063/1.5100835 \\ Submitted: 21 April 2019 - Accepted: 19 June 2019 • \\ Published Online: 17 July 2019
}

\author{
S. Capaccioli, ${ }^{1,2, a)}$ (D) K. L. Ngai, ${ }^{1,3, b)}$ S. Ancherbak, ${ }^{2}$ M. Bertoldo, ${ }^{4}$ G. Ciampalini, ${ }^{2}$ M. Shahin Thayyil, \\ and Li-Min Wang
}

\begin{abstract}
AFFILIATIONS
${ }^{1}$ CNR-IPCF, Dipartimento di Fisica, Largo Bruno Pontecorvo 3, I-56127, Pisa, Italy

²Dipartimento di Fisica, Università di Pisa, Largo Bruno Pontecorvo 3, I-56127, Pisa, Italy

${ }^{3}$ State Key Lab of Metastable Materials Science and Technology, and College of Materials Science and Engineering,

Yanshan University, Qinhuangdao, Hebei, 066004, China

${ }^{4}$ ISOF - CNR Area della Ricerca di Bologna, Via P. Gobetti 101, 40129 Bologna, Italy

${ }^{5}$ Department of Physics, University of Calicut, Kerala, India
\end{abstract}

Note: This paper is part of a JCP Special Topic on Chemical Physics of Supercooled Water.

a) simone.capaccioli@unipi.it. Tel.: +39-0502214537. Fax: +39-0502214333.

b) kia.ngai@pi.ipcf.cnr.it and kiangai@Yahoo.com. Tel.: +39-0502214322. Fax: +39-0502214333.

\begin{abstract}
Although by now the glass transition temperature of uncrystallized bulk water is generally accepted to manifest at temperature $T_{g}$ near $136 \mathrm{~K}$, not much known are the spectral dispersion of the structural $\alpha$-relaxation and the temperature dependence of its relaxation time $\tau_{\alpha, b u l k}(T)$. Whether bulk water has the supposedly ubiquitous Johari-Goldstein (JG) $\beta$-relaxation is a question that has not been answered. By studying the structural $\alpha$-relaxation over a wide range of temperatures in several aqueous mixtures without crystallization and with glass transition temperatures $T_{g}$ close to $136 \mathrm{~K}$, we deduce the properties of the $\alpha$-relaxation and the temperature dependence of $\tau_{\alpha, b u l k}(T)$ of bulk water. The frequency dispersion of the a-relaxation is narrow, indicating that it is weakly cooperative. A single Vogel-Fulcher-Tammann (VFT) temperature dependence can describe the data of $\tau_{\alpha, b u l k}(T)$ at low temperatures as well as at high temperatures from neutron scattering and $\mathrm{GHz}-\mathrm{THz}$ dielectric relaxation, and hence, there is no fragile to strong transition. The $T_{g}$-scaled VFT temperature dependence of $\tau_{\alpha, b u l k}(T)$ has a small fragility index $m$ less than 44, indicating that water is a "strong" glass-former. The existence of the JG $\beta$-relaxation in bulk water is supported by its equivalent relaxation observed in water confined in spaces with lengths of nanometer scale and having Arrhenius $T$-dependence of its relaxation times $\tau_{\text {conf }}(T)$. The equivalence is justified by the drastic reduction of cooperativity of the $\alpha$-relaxation in nanoconfinement and rendering it to become the JG $\beta$-relaxation. Thus, the $\tau_{\text {conf }}(T)$ from experiments can be taken as $\tau_{\beta, b u l k}(T)$, the JG $\beta$-relaxation time of bulk water. The ratio $\tau_{\alpha, b u l k}\left(T_{g}\right) / \tau_{\beta, b u l k}\left(T_{g}\right)$ is smaller than most glass-formers, and it corresponds to the Kohlrausch $\alpha$-correlation function, $\exp \left[-\left(t / \tau_{\alpha, b u l k}\right)^{1-n}\right]$, having $(1-n)=0.90$. The dielectric data of many aqueous mixtures and hydrated biomolecules with $T_{g}$ higher than that of water show the presence of a secondary $v$-relaxation from the water component. The $v$-relaxation is strongly connected to the a-relaxation in properties, and hence, it belongs to the special class of secondary relaxations in glass-forming systems. Typically, its relaxation time $\tau_{v}(T)$ is longer than $\tau_{\beta, b u l k}(T)$, but $\tau_{v}(T)$ becomes about the same as $\tau_{\beta, b u l k}(T)$ at sufficiently high water content. However, $\tau_{v}(T)$ does not become shorter than $\tau_{\beta, b u l k}(T)$. Thus, $\tau_{\beta, b u l k}(T)$ is the lower bound of $\tau_{v}(T)$ for all aqueous mixtures and hydrated biomolecules. Moreover, it is $\tau_{\beta, b u l k}(T)$ but not $\tau_{\alpha}(T)$ that is responsible for the dynamic transition of hydrated globular proteins.
\end{abstract}

Published under license by AIP Publishing. https://doi.org/10.1063/1.5100835 


\section{INTRODUCTION}

In 2011, we published a paper in this journal ${ }^{1}$ to resolve the controversy of glass transition and its temperature of amorphous water. The onset glass transition $T_{g}$ for vitreous water occurs at about $136 \mathrm{~K}$ for amorphous solid water (ASW) and hyperquenched glassy water (HGW) by differential scanning calorimetry (DSC) at a heating rate of $30 \mathrm{~K} / \mathrm{min}^{2,3}$ The low density amorph (LDA) made by isothermal decompression of very high-density amorphous ice at $140 \mathrm{~K}$ from 1.1 to $0.006 \mathrm{GPa}$ shows a glass-liquid transition endotherm with an onset temperature $T_{g}$ of $\sim 137$ (140) $\mathrm{K}$ at the same heating rate. ${ }^{4-6}$ The controversy came from the question raised on the validity of $T_{g} \approx 136 \mathrm{~K}$ for water by suggesting that the feature observed by DSC of ASW is not the $T_{\mathrm{g}}$ endotherm but a "shadow- $T_{\mathrm{g}}$ peak" or "sub- $T_{g}$ peak" appearing in the $0.7 T_{\mathrm{g}}-0.8 T_{\mathrm{g}}$ range in the DSC scan, ${ }^{7-11}$ and the alternative proposal that the glass transition in water is an order-disorder transition that occurs within the range from 150 to $250 \mathrm{~K}$, ${ }^{12}$ by Angell and co-workers. Recently, Angell no longer challenged that the $T_{g}$ of bulk water is equal to $136 \mathrm{~K}$, and the acceptance of $T_{g} \approx 136 \mathrm{~K}$ for water was made explicit in the publication by 16 authors ${ }^{13}$ including Angell. Also noted in this reference is that LDA, HGW, and ASW are the same material. The controversy can also be reconciled by the considerations given in Refs. 1 and 14 of the dynamics of water that the deduced $T_{g}$ of water cannot be anywhere but near the generally accepted value of $T_{g} \sim 136 \mathrm{~K}$. These considerations include the following: (i) some glass-forming aqueous mixtures with $T_{g}$ approaching the putative $T_{g}=136 \mathrm{~K}$ of water from above and below, (ii) water confined in nanometer spaces, and (iii) bulk water at temperatures above the homogeneous nucleation temperature $\sim 235 \mathrm{~K}$. We are not the first ones to use some appropriate aqueous solutions to approximate the $\alpha$-relaxation of water. This was done before by Angell and co-workers, ${ }^{9,10,15}$ and by Sokolov and co-workers. ${ }^{16}$ The reason why some of these aqueous solutions were chosen is because their glass transition temperatures are close to $136 \mathrm{~K}$ from dielectric and calorimetry. Examples are $\mathrm{N}_{2} \mathrm{H}_{2}$ and $\mathrm{LiCl}$ water solutions having calorimetric $T_{g}$ close to $136 \mathrm{~K}$ of water, as shown in Fig. 1 in the paper by Angell. ${ }^{12}$ Both water and the solution participate in the structural $\alpha$-relaxation of the aqueous solutions, and the observed $\alpha$-relaxation time $\tau_{\alpha}$ is necessarily different from that of pure water.

The considered relaxation times from the data range over 15 decades from $10^{-12}$ to $10^{3} \mathrm{~s}$. Assisted by the various features in the isothermal spectra and theoretical interpretation, these considerations enable us to conclude by inference from (i) and (ii) that relaxation of uncrystallized water is weakly cooperative. In other words, the degree of dynamic heterogeneity and the length-scale of its structural $\alpha$-relaxation are small. The exponent $\beta_{K}$ of its Kohlrausch stretched exponential correlation function is not much less than the value of one, and hence, the deviation from exponential time decay is slight. This deduction is supported by the interpretation of dielectric relaxation of water in the range from $\mathrm{GHz}$ to beyond $\mathrm{THz}$ at temperatures above and below room temperature. ${ }^{17}$ The temperature dependence of its $\alpha$-relaxation time is non-Arrhenius, and the corresponding $T_{g}$-scaled temperature dependence has small fragility index $m$, likely less than 44 at $T_{g}$, and hence water is a "strong" glass-former. Again from (i) and (ii), we deduced that water has not only the structural $\alpha$-relaxation but also the Johari-Goldstein (JG) $\beta$-relaxation. The deduction is consistent with the existence of a secondary (strongly connected with the $\alpha$-relaxation in properties) relaxation in any glass-former. ${ }^{18-21}$ Secondary relaxation of this kind was called the JG $\beta$-relaxation to distinguish from other secondary relaxations devoid of the connections.

The existence of the JG $\beta$-relaxation of bulk water and the activation energy of about $48 \mathrm{~kJ} / \mathrm{mol}$ in the Arrhenius $T$-dependence of its relaxation time $\tau_{\beta, b u l k}(T)$ below $T_{g} \sim 136 \mathrm{~K}$ were brought out for the first time in Refs. 1 and 14 from considerations of aqueous mixtures. For each mixture, the primary $a$-relaxation and a secondary process were observed below. The $a$-relaxation is due to the cooperative motion of water and solute molecules and responsible for the thermal glass transition of the mixture. The secondary process is due to the local motion of noncrystallized water. The water molecules contributing to this process do not move cooperatively with solute molecules although its relaxation time depends on the solute and its concentration. This process was named the $v$-process

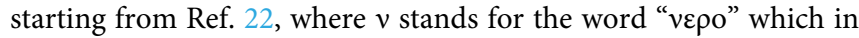
Greek means "water." Furthermore, in Refs. 1, 14, and 23, it was shown that the relaxation times $\tau_{v}(T)$ of the $v$-process in aqueous mixtures and hydrated proteins with sufficiently large water content and $\tau_{\text {conf }}(T)$ in nanoconfined water with Arrhenius temperature dependence are practically the same as the JG $\beta$-relaxation time $\tau_{J G, b u l k}(T)$ of bulk water (see Figs. 4 and 7 of Ref. 14 and Fig. 10 of Ref. 19). The results are consistent with that expected from the Coupling Model $(\mathrm{CM})^{15,18}$ from the small degree of cooperativity of bulk water and further reduction in the cooperativity lengthscale in nanoconfinement or by the high water content in aqueous mixtures.

Before Refs. 1, 14, and 20 and even earlier in Refs. 22 and 25, the JG $\beta$-relaxation of bulk water was hardly considered by others in the water research community, although the existence of secondary relaxation in aqueous mixture is well known, ${ }^{26-29}$ and the Arrhenius relaxation found in nanoconfined water was interpreted differently. $^{27,29}$ We thank Cerveny et al. ${ }^{30}$ for mentioning our interpretation of the Arrhenius relaxation of nanoconfined water by the JG $\beta$-relaxation of water and the difference from their own interpretation. It is worth citing their statement in this regard: "Interestingly, and in agreement with these results, other workers ${ }^{64}$ argued that the process showing a crossover to Arrhenius behavior at low temperatures has the characteristic of a Johari-Goldstein relaxation (JG)." Currently, the JG $\beta$-relaxation of water is widely appreciated by others. It was mentioned by the same name, Johari-Goldstein $\beta$-relaxation of water, in Refs. 31 and 32. Our 2011 results on the JG $\beta$-relaxation of water was agreed upon by Swenson and Cerveny in their review" by the statement "Since this low-temperature $\beta$-relaxation is universal for all systems at high water content it is possible that it can be considered as an intrinsic $\beta$-relaxation of supercooled water, including supercooled bulk water." The JG $\beta$-relaxation of bulk water should be faster than the $v$-relaxation in aqueous mixtures, hydrated proteins at low or high water content, and nanoconfined water of any size. Hence, $\tau_{\beta, b u l k}(T)$ is the lower bound of $\tau_{v}(T)$ for all $T$, which is the follow-up conclusion we made in Refs. 1 and 13. This supplementary result was fully appreciated by Panagopoulou et al., and they verified this prediction by their data of hydrated proteins.

The separation in time scale of the a-relaxation and the $\beta$-relaxation of water is small at a low temperature near $T_{g}$ (see Fig. 4 of Ref. 1 and Fig. 1 of this paper to be presented later), consistent 
with the $\mathrm{CM}$ equation (1) (see later in Sec. III A), and $\beta_{K}$ is slightly smaller than 1 . It becomes even closer to one at high temperatures in the range from 275 to $350 \mathrm{~K}$ as shown by the analysis and interpretation of the $\mathrm{GHz}-\mathrm{THz}$ dielectric data ${ }^{35}$ in conjunction with the neutron scattering data ${ }^{36}$ by using the coupling model.

In this paper, we go deeper and further in characterizing and interpreting the $\alpha$-relaxation and the $\beta$-relaxation of water and their relationship, the connection to the fast relaxation observed in nanoconfined water, and the $v$-relaxation in aqueous mixtures and in hydrated biomolecules. The study is enriched by our own new studies of aqueous mixtures of hydrophilic substances of various kinds, as well as the hydrated proteins and biomolecules, with water content $C_{\mathrm{W}}$ or hydration level $h_{\mathrm{W}}$ (defined as the ratio between the mass of hydration water (HW) over the mass of protein sample) ranging from low to high. In conjunction with experimental data from the literature, the new experimental data verify that the $v$ relaxation is the precursor of the structural $\alpha$-relaxation in aqueous mixtures and hydrated proteins with strong connections between their properties, like the special class of $\beta$-relaxation in many kinds of glass-formers. ${ }^{18-21}$ These connections include the following: (i) the change of $\tau_{v}(T)$ from Arrhenius below $T_{g}$ to a stronger $T$-dependence above $T_{g}$, (ii) the separation of the $v$-relaxation from the a-relaxation at $T_{g}$ measured by $\left[\log \left(\tau_{\alpha}\left(T_{g}\right)\right)-\log \left(\tau_{v}\left(T_{g}\right)\right)\right]$ is proportional to the degree of nonexponentiality of the a-relaxation, and (iii) the dielectric strength of the $v$-relaxation changes to a stronger $T$-dependence on crossing $T_{g}$ from below. At a sufficiently high content of water $C_{\mathrm{W}}$ in aqueous mixtures and high hydration level $h$ in hydrated biomolecules, we find that the $v$-relaxation times, $\tau_{v}(T)$, are either close to or identical to $\tau_{\text {conf }}(T) \approx \tau_{\beta, b u l k}(T)$ of water in many different systems. This is a ubiquitous property of the $v$-relaxation at high $C_{\mathrm{W}}$ or $h$. Thus, in the form of the $v$-relaxation, the JG $\beta$-relaxation of water shows up in aqueous mixtures and hydrated biomolecules at high $C_{\mathrm{W}}$ or $h$. Since $T_{g}$ of many aqueous mixtures and hydrated biomolecules is considerably higher than $136 \mathrm{~K}$, this means that the JG $\beta$-relaxation of water persists therein to higher temperatures than possible in water, and it responds to the glass transition effected by the structural $\alpha$-relaxation by changing to a stronger $T$-dependence above $T_{g}$. All the properties mentioned in this section will be demonstrated by experimental data in Secs. III-V.

In addition, the results in hydrated proteins and biomolecules help confirm that there is a change in the temperature dependence of the $v$-relaxation time $\tau_{v}(T)$ at $T_{g}$ in response to vitrification at $T_{g}$. This effect is observed as a change in temperature dependence of the mean square displacement (MSD) by Mössbauer spectroscopy ${ }^{3}$ or neutron scattering. ${ }^{38-40}$ There is yet another change in the MSD at the higher dynamic transition temperature $T_{d}$ where $\tau_{v}\left(T_{d}\right)$ or $\tau_{\beta}\left(T_{d}\right)$ starts to enter the time scale or time window of the spectrometer of Mössbauer spectroscopy ${ }^{40}$ and neutron scattering IN16, IN10, IN13, and IN5 or IN6. ${ }^{41-43}$ The shorter the time scale of the spectrometer, the higher the $T_{d}$. If the hydration level is high, $\tau_{v}\left(T_{d}\right)$ approaches a value depending mainly on the spectrometer time scale and affected very little by the protein structure.

\section{EXPERIMENTAL DETAILS}

We report new measurements and data on the dynamics of water in glass-forming mixtures with mono-, di-, and poly-saccharides (deoxyribose, fructose, glucose, sucrose, trehalose, and glycogen) and on a hydrated protein ribonuclease A (RNase A). The glycogen $\left(\mathrm{M}_{\mathrm{w}}=270 \mathrm{kDa}\right.$, polydispersity 1.2) was kindly supplied by "Aziende Chimiche Angelini Francesco ACRAF s.p.a." This was obtained from molluscs Mytilus edilus and Mytilus gallus provincialis in a form substantially free of nitrogenous compounds and reducing sugars. For dielectric measurements, water-glycogen mixtures with $\mathrm{C}_{\mathrm{W}}=23 \mathrm{wt}$. $\%, 34 \mathrm{wt}$. $\%(h=0.30,0.52)$ were prepared by solution casting and drying of 90 wt. \% water-glycogen solution on the electrodes in nitrogen atmosphere. The other hydrophilic solutes and RNAse were obtained from Sigma-Aldrich and used as received. The humidity content of each sample before the hydration procedure was estimated by thermogravimetry. Distilled and de-ionized water (electric conductivity lower than $18.3 \mu \mathrm{s} \mathrm{m}^{-1}$ ) was prepared by an ultrapure water distiller (Millipore, MILLI-Q Lab). Both mixing of components and loading of the sample holder were performed in controlled atmosphere (dry nitrogen). RNase powder was hydrated and compacted by using the same procedures used for biomolecules in Refs. 44-46.

Data have been obtained by dielectric spectroscopy in the frequency range from $10 \mathrm{mHz}$ to $3 \mathrm{GHz}$, by means of a combination of dielectric response analysis and coaxial reflectometry using the Novocontrol dielectric analyzer and the Agilent network analyzers $8722 \mathrm{D}$ and $8753 \mathrm{ES}$. Dielectric study was carried out by using sealed cells, whose temperature was varied in a wide range, spanning from the deep glassy state to ambient temperature, following isothermal steps after an appropriate equilibration time. For further details on the experimental technique and on the dielectric spectra analysis, see Ref. 42 .

\section{ASW, NANOCONFINED WATER, AND WATERLIKE SOLUTIONS}

\section{A. Lower temperatures}

In the previous paper, ${ }^{1}$ we approximately locate the $T_{g}$ as well as the $\alpha$-relaxation times $\tau_{\alpha, b u l k}(T)$ of water by several methods. One method is to use the structural $\alpha$-relaxation time $\tau_{\alpha}(T)$ of aqueous mixture with 22.5 and $30.3 \mathrm{~mol}$. \% of ethylene glycol (EG) $\left(\mathrm{C}_{\mathrm{W}}\right.$ $=50 \%, 40 \%)^{47}$ and water solution of hydrazine with $26.5 \mathrm{~mol}$. \% of hydrazine $\mathrm{N}_{2} \mathrm{H}_{4}$. Dielectric spectra of 22.5 and 30.3 mol. \% EG water mixtures can be found in Figs. S2 and S3 of the supplementary material. As discussed in Ref. 1, $\tau_{\alpha}(T)$ in the water solution of $22.5 \mathrm{~mol}$. \% of EG is slightly longer than $\tau_{\alpha, b u l k}(T)$. On the other hand, $\tau_{\alpha}(T)$ of the water solution of $26.5 \mathrm{~mol}$. \% of $\mathrm{N}_{2} \mathrm{H}_{2}$ is slightly shorter than $\tau_{\alpha, b u l k}(T)$. In fact, at $136 \mathrm{~K}, \tau_{\alpha}(T)$ of the $22.5 \mathrm{~mol}$. \% EG solution is slower than $\tau_{\alpha, b u l k}(T)=33 \mathrm{~s}$ of amorphous solid water (ASW) represented by the large filled triangle, ${ }^{48}$ while $\tau_{\alpha}(T)$ of the 26.5 mol. \% $\mathrm{N}_{2} \mathrm{H}_{2}$ solution is a bit faster (see Fig. 1 which contains more data than the original figure in Ref. 1 ).

We have added new $\tau_{\alpha}(T)$ data of $\left(\mathrm{H}_{2} \mathrm{O}\right)_{0.88}(\mathrm{LiCl})_{0.12}$ taken from the work of Nakanishi et al. ${ }^{16}$ in Fig. 1. $\tau_{\alpha}(T)$ of the $\mathrm{LiCl}$ solution are faster than that of the $22.5 \mathrm{~mol}$. \% EG solution but still a bit slower than that of $26.5 \mathrm{~mol} . \% \mathrm{~N}_{2} \mathrm{H}_{2}$ solution. The dielectric permittivity and loss tangent of hyperquenched glassy water (HGW) were measured for fixed frequencies of 1 and $10 \mathrm{kHz}$ by Johari et al. ${ }^{48}$ The dielectric $\tan (\delta)$ peak of ASW at $1 \mathrm{kHz}$ found at $\sim 157 \mathrm{~K}$, corresponding to $\tau_{\alpha}=10^{-3.8} \mathrm{~s}$, is included in Fig. 1 and shown by the large closed 


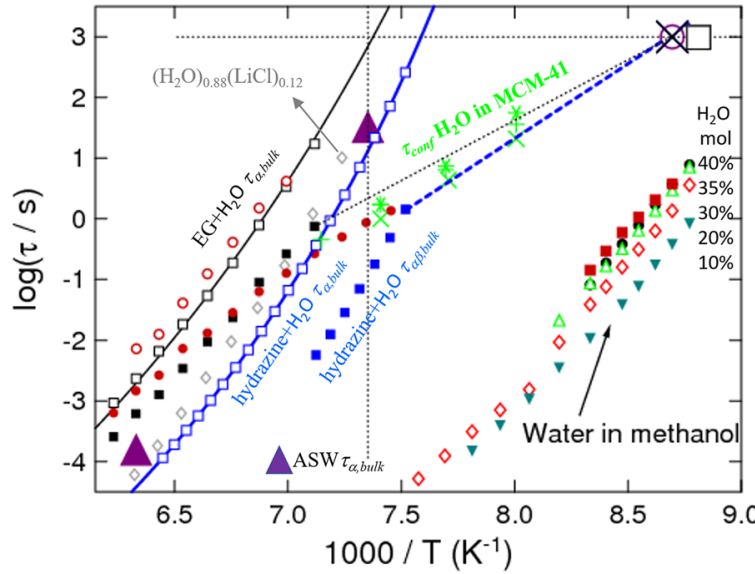

FIG. 1. Estimating the $\tau_{\alpha, b u l k}(T)$ of bulk water by $\tau_{\alpha}(T)$ of water in aqueous mixture with 22.5 (black open squares) and 30.3 (red open circles) mol. \% of EG, water solution of hydrazine with $26.5 \mathrm{~mol}$. \% of $\mathrm{N}_{2} \mathrm{H}_{4}$ (blue open squares), and $\left(\mathrm{H}_{2} \mathrm{O}\right)_{0.88}(\mathrm{LiCl})_{0.12}$ (gray open diamonds). The two large filled triangles are $\tau_{\alpha, b u l k}(T)$ at $136 \mathrm{~K}$ from the DSC endotherm of ASW and corresponding to $1 \mathrm{kHz}$ dielectric $\tan \delta$ data (see text). Dielectric $\tau_{\text {conf }}(T)$ of water confined in molecular sieves MCM-41 with pore diameter $2.14 \mathrm{~nm}$ at hydration levels $12 \mathrm{wt} . \%$ (green crosses) and $22 \mathrm{wt}$ \% (green stars), by adiabatic calorimetry of water confined within nanopores of MCM-41 (large purple circled cross), and water confined within silica pores (large black square). Blue and black lines are Vogel-Fulcher-Tammann (VFT) fits to $\tau_{\alpha}(T)$ of the water/hydrazine mixture. Closed blue squares are the primitive relaxation times of the water/hydrazine mixture. Dashed lines suggest the Arrhenius dependence of $\tau_{\beta, b u l k}(T)$ in the glassy state for two mixtures. The data on the right corner are relaxation times of water in mixtures with methanol. Water mole fractions: $10 \%, 20 \%, 30 \%, 35 \%$, and $40 \%$ from bottom to top.

triangle at the corner of the bottom. The $\tan (\delta)$ data were taken on the nanometer-size water droplets coexisting with cubic ice particles of about the same size at temperatures in the 150-180 K range. ${ }^{4}$ The ASW $\tau_{\alpha}$ at $T=157 \mathrm{~K}$ from $\tan (\delta)$ measurement lies close to the $\tau_{\alpha}(T)$ of the $\mathrm{LiCl}$ solution and the $\mathrm{N}_{2} \mathrm{H}_{2}$ solution, and suggests that $\tau_{\alpha}(T)$ of the two solutions are good estimates of the $\tau_{\alpha, b u l k}(T)$ of water in the extended temperature region if water is prevented from being crystallized. Moreover, this implies that the temperature dependence of $\tau_{\alpha, \text { bulk }}(T)$ is also super-Arrhenius or Vogel-FulcherTammann like that of the $\mathrm{LiCl}$ and the $\mathrm{N}_{2} \mathrm{H}_{2}$ solution. The dielectric loss spectra of the 22.5 mol. \% EG solution show an excess loss not accounted for by the main $\alpha$-loss peak, which was identified with the contribution from the $v$-relaxation, and its relaxation times $\tau_{v}(T)$ of the solution itself determined by fits to the spectra ${ }^{1}$ are shown by the closed red circles in Fig. 1 . These values of $\tau_{v}(T)$ can be considered as approximate estimates of the $\tau_{\beta, b u l k}(T)$ of water. The $v$ relaxation of water in solutions of hydrazine was not resolved in the dielectric spectra, and its relaxation time $\tau_{v}$ is approximated by the primitive relaxation time $\tau_{0}$ calculated by the Coupling Model (CM) equation,

$$
\tau_{\alpha}=\left[t_{c}^{-n} \tau_{0}\right]^{1 /(1-n)} \approx\left[t_{c}^{-n} \tau_{v}\right]^{1 /(1-n)},
$$

where $t_{c}=1-2$ ps for van der Waals glass-formers but shorter than $0.2 \mathrm{ps}$ for hydrogen-bonded glass-formers, ${ }^{21}$ and $(1-n)$ is the fractional exponent in the Kohlrausch function used to fit the dielectric a-loss peak of the 26.5 mol. $\% \mathrm{~N}_{2} \mathrm{H}_{2}$ solution. In many different kinds of glass-forming systems, the approximate agreement between the JG $\beta$-relaxation time and $\tau_{0}$ had been verified.

Another method to observe the dynamics of uncrystallized water is confinement in various spaces with nanometer size. There are a number of such dielectric studies of water confined in different media including vermiculite clay, ${ }^{27}$ molecular sieves, ${ }^{50,51}$ MCM-41 $(21 \AA),{ }^{52,53}$ graphite oxide, ${ }^{30}$ calcium silicate hydrate gel, ${ }^{54}$ and silica gel. ${ }^{55}$ The experiments were reviewed by Swenson and Cerveny. ${ }^{31}$ All observed a fast relaxation with the observed relaxation times $\tau_{\text {conf }}$ having Arrhenius temperature dependence and are as short as $\tau_{v}(T)$ of the $22.5 \mathrm{~mol}$. \% EG solution or the primitive relaxation time $\tau_{0}(T)$ of the $26.5 \mathrm{~mol}$. $\% \mathrm{~N}_{2} \mathrm{H}_{2}$ solution, which are approximately $\tau_{\beta, b u l k}$ of water as mentioned above. The link of the $\tau_{\text {conf }}$ to the JG $\beta$-relaxation time $\tau_{\beta, \text { bulk }}$ of water and of aqueous mixture was brought out for the first time in Refs. 1, 14, and 23.

Such dielectric data of $\tau_{\text {conf }}$ found by Hedstrom et al. ${ }^{52}$ in water confined in molecular sieves MCM- 41 with pore diameter $2.14 \mathrm{~nm}$ at hydration levels 12 wt. \% (green crosses) and 22 wt. \% (green stars), and in silica gels by Cammarata et al. ${ }^{55}$ are shown as a function of reciprocal temperature in Fig. 1. Only part of the complete set of data in Refs. 52 and 55 at long $\tau_{\text {conf }}(T)$ and low $T$ are shown in Fig. 1 because only these are relevant for the present discussion of water. The shorter $\tau_{\text {conf }}(T)$ at higher $T$ have already merged with the a-relaxation of water. Oguni and co-workers have measured the relaxation of water confined within $1.2,1.6$, and $1.8 \mathrm{~nm}$ nanopores of MCM-41 by adiabatic calorimetry, and the fastest relaxation having a relaxation time of $10^{3} \mathrm{~s}$ at $T=115 \mathrm{~K}$ is shown by the large purple circled cross in Fig. $1 .^{56}$ The large open square in Fig. 1 indicates the value of $1000 / T$ at which $\tau_{\text {conf }}=10^{3} \mathrm{~s}$ was found in water confined within silica pores by adiabatic calorimetry. ${ }^{57}$ It can be seen from Fig. 1 that $\tau_{\text {conf }}$ has time scale and temperature dependences unlike $\tau_{\alpha}$ of water and instead like that expected for the $\tau_{\beta, b u l k}(T)$ of water. This is readily understandable in view of the further reduction of cooperativity and length-scale of the $\alpha$-relaxation of water when confined in nanometer space, and thus, effectively $\tau_{\alpha}$ is reduced to $\tau_{\beta, b u l k}(T)$. Thus, we have the $\tau_{\beta, b u l k}(T)$ of water from the values of $\tau_{\text {conf }}(T)$ obtained by adiabatic calorimetry and dielectric relaxation. The value of $\tau_{\alpha}$ is $33 \mathrm{~s}$ at $136 \mathrm{~K}$ for ASW, and $\tau_{\beta, b u l k}(T)=\tau_{\text {conf }}(T)$ has approximately the value of $1 \mathrm{~s}$ at the same temperature (see Fig. 1). By applying the CM equation now written alternatively as

$$
n \approx\left(\log \tau_{\alpha, b u l k}-\log \tau_{\beta, b u l k}\right) /\left(\log \tau_{\alpha, b u l k}+12.7\right),
$$

we get an estimate of the coupling parameter $n$ of ASW of 0.10 , verifying that cooperativity of water is small. This value is consistent with the small values of $n=0.16,0.16$, and 0.19 at temperatures of $154 \mathrm{~K}, 150 \mathrm{~K}$, and $146 \mathrm{~K}$, respectively, of the $26.5 \mathrm{~mol} . \% \mathrm{~N}_{2} \mathrm{H}_{2}$ solution determined directly by fitting the dielectric loss spectra, ${ }^{1}$ and the consistency throws another support for $\tau_{\alpha}(T)$ of the $26.5 \mathrm{~mol}$. \% $\mathrm{N}_{2} \mathrm{H}_{2}$ solution being close to $\tau_{\alpha, b u l k}(T)$ of water.

The relation of $\tau_{\text {conf }}$ to $\tau_{\beta, \text { bulk }}(T)$ of water in aqueous mixture with 22.5 and $30.3 \mathrm{~mol}$. \% of EG and water solution of hydrazine with 26.5 mol. \% of $\mathrm{N}_{2} \mathrm{H}_{4}$ is brought out, respectively, by the black dotted and red and blue dashed lines connecting $\tau_{\text {conf }}$ of water confined in silica pores or MCM-41 from adiabatic calorimetry to the longest $\tau_{v}$ in Fig. 1. These lines also suggest plausible temperature 
dependence of $\tau_{\beta}$ in the glassy state of the three aqueous mixtures, which are close to the $\tau_{\beta}$ of water as can be seen by these lines lying close to the values of $\tau_{\text {conf }}(T)$ of water nanoconfined in MCM $-41^{52}$ and silica gels ${ }^{55}$ and identifiable with $\tau_{\beta, \text { bulk }}(T)$ of water with an activation energy of about $48 \mathrm{~kJ} / \mathrm{mol}$.

Usually, water relaxes faster than most carbon containing molecules, and the observed $\tau_{v}$ of the water solution is slower than $\tau_{\beta, b u l k}$ of water. However, there are exceptions including 1-propanol and methanol. We have considered the effect of 1-propanol on water before. ${ }^{1}$ The effects of mixing methanol to water on $\tau_{v}$ are more dramatic. ${ }^{58}$ As shown in Fig. 1, $\tau_{v}$ are many orders of magnitude faster and increase with decreasing water mole fractions from $40 \%$ down to $10 \%$. Thus, water behaves normally when it is the faster component as in many ordinary binary mixtures of non-hydrogen-bonded liquids. ${ }^{20,21}$

\section{B. Dynamics of water at all temperatures}

Dielectric measurements of bulk water at high frequencies above $1 \mathrm{GHz}$ and temperatures higher than $T_{H}=235 \mathrm{~K}$ published by several groups are consistent with each other ${ }^{59-62}$ and with the recent

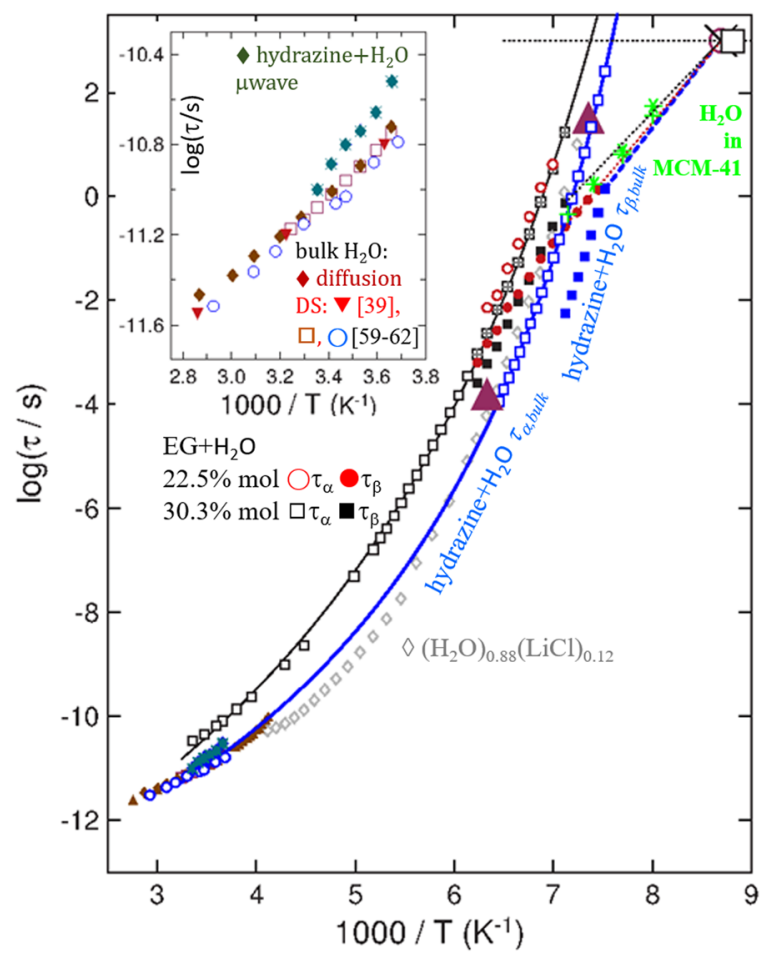

FIG. 2. Logarithm of relaxation times vs reciprocal temperature for different relaxation processes and in different systems represented by the same symbols as in

1. At the left bottom are dielectric relaxation times $\tau_{D}(T)$ for bulk water and the relaxation times deduced from diffusion data, just above the relaxation times from microwave measurements of solution of hydrazine. The data with relaxation times shorter than $1 \mathrm{~ns}$ are enlarged in the inset, where the new gigahertz to terahertz dielectric data in Ref. 35 are added and represented by red inverted triangles. The figure is an updated version of Fig. 7 of Ref. 1, and the same symbols are used to represent the data. measurements made by Lunkenheimer et al. ${ }^{35}$ Their dielectric relaxation times $\tau_{D}$ of less than $10^{-10} \mathrm{~s}$ and superposing on each other are presented at the very bottom in Fig. 2 and magnified in the inset where three data points (inverted red triangles) are taken from the work of Lunkenheimer et al. The relaxation times shown by stars and longer than $\tau_{D}$ of water are high frequency dielectric relaxation times of solutions of polyvinyl alcohol (PVOH), polyvinylpyrrolidone (PVP), and ethylene glycol (EG) with 90, 80, and 40 wt. \% of water, respectively. ${ }^{63}$ The values are higher but still close to $\tau_{D}$ of bulk water. The blue line is the Vogel-Fulcher-Tammann-Hesse (VFTH) fit to the $\tau_{\alpha}(T)$ data of the $26.5 \mathrm{~mol}$. hydrazine solutions published by Minoguchi et al. ${ }^{7,910}$ This VFTH dependence of $\tau_{\alpha}(T)$ as well as the $\tau_{\alpha}(T)$ data of $\left(\mathrm{H}_{2} \mathrm{O}\right)_{0.88}(\mathrm{LiCl})_{0.12}$ from the work of Nakanishi et al. ${ }^{16}$ smoothly joins to the $\tau_{D}(T)$ of water at higher temperatures. Thus, there is no need to invoke the fragile-strong transition of water as concluded also by Nakanishi et al. since the $\tau_{\alpha}(T)$ of these two solutions of water are supposed to be approximately the $\tau_{\alpha}(T)$ of water.

The temperature dependence of $\tau_{D}$ of water at higher temperatures is Arrhenius and has an activation energy of about 17-18 $\mathrm{kJ} / \mathrm{mol}$, comparable to that determined by viscosity and IXS. ${ }^{1}$ The intense dielectric loss peak of bulk water observed in the gigahertz-terahertz range ${ }^{35}$ is almost Debye-like with correlation function that is nearly an exponential function of time. This was interpreted recently as the a-relaxation having the Kohlrausch correlation function that is nearly an exponential function of time, i.e., the Kohlrausch exponent $(1-n)$ is close to 1 , consistent with $(1-n)=0.90$ determined in connection with the data in Fig. 1 at low temperatures. The high frequency power law on the high frequency side of the $\alpha$-loss peak was interpreted as due to the combination of the unresolved $\beta$-relaxation of water and the caged dynamics contribution.

\section{AQUEOUS MIXTURES WITH Tg APPRECIABLY HIGHER THAN WATER}

Hydrazine and EG are small enough molecules chosen such that their water solutions have $T_{g}$ and $\tau_{\alpha}(T)$ approaching those of ASW and uncrystallized water at sufficiently high molar fraction $X_{w}$ of water. Also, the resolved secondary relaxation has its $\tau_{v}(T)$ approximately the same as the $\tau_{\beta, b u l k}(T)$ of water. The situations of water solutions of larger molecules are different especially if the molar fraction of water is not high. The $T_{g}$ is higher than $136 \mathrm{~K}$, and $\tau_{v}(T)$ of the $v$-relaxation contributed by water is much longer than $\tau_{\beta, b u l k}(T)$ of water. The differences are demonstrated by the temperature dependences of $\tau_{\alpha}(T)$ and $\tau_{v}(T)$ in water solutions of oligomers of EG, $j \mathrm{EG}$ with $j$ ranging from 2 to 6 all with $\mathrm{C}_{\mathrm{W}}=35$ wt. $\%$ of water, and they are compared with those of the 40 wt. \% (same as $30.3 \mathrm{~mol}$. \%) of EG (red open and closed circles). The $\tau_{v}(T)$ of the oligomers are longer than that of EG at the same temperature, associated with the increase in $T_{g}$. Notwithstanding, a change from Arrhenius $T$-dependence for $T<T_{g}$ of $\tau_{v}(T)$ to a stronger one for $T<T_{g}$ is found for all oligomers at the crossing temperature $T_{c}$ near $T_{g}$ as indicated by the vertical arrows in Fig. 3 and $T_{g}$ is operationally defined as the temperature at which $\tau_{\alpha}\left(T_{g}\right)=10^{3} \mathrm{~s}$. The dielectric strength $\Delta \varepsilon_{v}(T)$ also changes its temperature dependence on crossing $T_{c}$ near $T_{g}{ }^{24}$ This behavior of $\tau_{v}(T)$ and $\Delta \varepsilon_{v}(T)$ is one of 


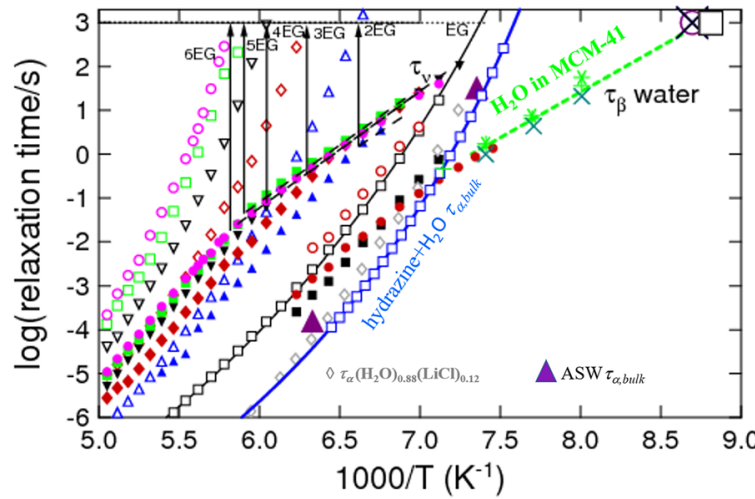

FIG. 3. Temperature dependence of the dielectric relaxation time $\tau_{\alpha}(T)$ (open symbols) and $\tau_{v}(T)$ (corresponding closed symbols) of mixtures of $35 \mathrm{wt} . \%$ of water with various ethylene glycol oligomers $6 E G, 5 E G, 4 E G, 3 E G$, and $2 E G$. Some of the data of $\tau_{v}(T)$ (closed symbols) overlap and cannot be easily resolved. For this reason, we use the dashed lines to indicate the Arrhenius temperature dependences assumed by $\tau_{v}(T)$ of the mixtures starting approximately at temperatures slightly below $T_{\mathrm{g}}$ of the mixtures defined by $\tau_{\alpha}(\mathrm{Tg})=10^{3} \mathrm{~s}$ located by the vertical arrows drawn. The change in temperature dependence of $\tau_{v}$ at $T_{\mathrm{g}}$ is similar to that in the JG $\beta$-relaxation of the faster component in nonaqueous mixtures. The other symbols and lines are the same as in Fig. 1. Dielectric spectra of $j E G$ mixtures can be found in Refs. 64-66.

the indicators of the strong connection or coupling between the $v$ relaxation and the structural $\alpha$-relaxation. By contrast, others interpreted the behavior as a decoupling of the $\beta$-like water relaxation from the structural $\alpha$-relaxation. ${ }^{31}$ We think the term "decoupling" used is inappropriate. This property is general and found for a special class of secondary relaxation in many glass-formers we called the JG $\beta$-relaxations, ${ }^{18-21}$ and it is predicted by the CM equation (1). ${ }^{21}$ In other words, the change in temperature dependence of $\tau_{\alpha}(T)$ at $T_{g}$ caused by vitrification has to be followed by $\tau_{v}(T)$ because of Eq. (1).

The ratio $\tau_{\alpha}\left(T_{g}\right) / \tau_{v}\left(T_{g}\right)$ increases with the size $j$ of the oligomer or $T_{g}$. It is intuitively reasonable to expect stronger intermolecular coupling of water to oligomer of larger size and less mobility or larger coupling parameter $n$ in the framework of the CM. Thus, the trend of $\tau_{\alpha}\left(T_{g}\right) / \tau_{v}\left(T_{g}\right)$ increasing with $T_{g}$ of the oligomers is consistent with the prediction from the CM equation rewritten as $\log \left[\tau_{\alpha}\left(T_{g}\right) / \tau_{v}\left(T_{g}\right)\right]=n\left[\tau_{\alpha}\left(T_{g}\right)+\log t_{c}\right]$ and by the corresponding increase in $n$.

To gain more insights into the $v$-relaxation and its relation to the $\alpha$-relaxation in water solutions, we have renewed studies of solutions where the hydrophilic solutes that have not been studied before are the glucose, sucrose, deoxyribose, trehalose, fructose, and glycogen in the carbohydrate family. In these dielectric studies, the dynamics are revealed by isothermal dielectric spectra over broad frequency range. Instead of changing the molecular size at constant wt. \% of water as in Fig. 3 for the $j \mathrm{EG}$ oligomers, we study the same solute but vary the content of water. For the purpose of this paper, we present only the relaxation times $\tau_{\alpha}(T)$ and $\tau_{v}(T)$ deduced from the relaxation spectra but not the spectra themselves. The data of $\tau_{\alpha}(T)$ and $\tau_{v}(T)$ in water solutions of glucose with $C_{w}=15,26,30$, and 38 wt. \% of water are presented in Fig. 4.

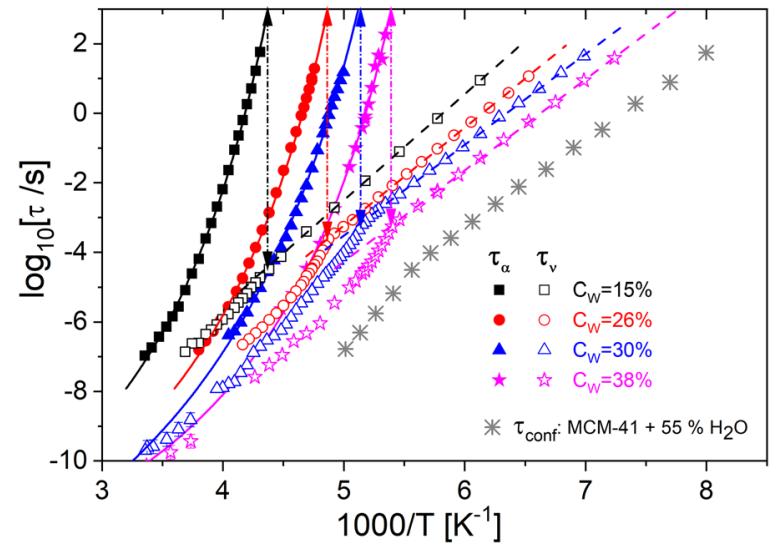

FIG. 4. Relaxation times $\tau_{\alpha}(T)$ and $\tau_{v}(T)$ in water solutions of glucose with $C_{w}=15,26,30$, and 38 wt. $\%$ of water plotted vs reciprocal temperature, together with $\tau_{\text {conf }}(T)$ (green asterisks) of water confined in MCM- $411^{52}$ Vertical arrows locate $1000 / T_{g} s$, where $T_{g} s$ is the glass transition of the solution. Continuous lines are VFT fits to $\tau_{\alpha}(T)$, and dashed lines are Arrhenius law fits to $\tau_{v}(T)$ below $T_{\mathrm{gs}}$.

Dielectric spectra of these systems can be found in Figs. S5-S8 of the supplementary material. The $\tau_{v}(T)$ of the glucose water solutions are longer than $\tau_{\beta}(T)$ of water estimated by the $\tau_{\text {conf }}(T)$ of water confined in MCM-41 ${ }^{52}$ in Fig. 4 , where the data of $\tau_{\text {conf }}(T)$ at higher temperatures than in Figs. 1 and 2 are shown. This is in contrast to $\tau_{v}(T) \approx \tau_{\text {conf }}(T) \approx \tau_{\beta, \text { bulk }}(T)$ in the case of the 30.3 mol. \% water solutions of EG and the $26.5 \mathrm{~mol}$. \% water solution of hydrazine (see Fig. 1). The change is due to glucose being structurally more complex and has $T_{g}$ higher than EG and hydrazine. The increase in $\tau_{v}(T)$ with the decrease in $C_{w}$ is much less than that of $\tau_{\alpha}(T)$. The arrows locate the $T_{g} \mathrm{~s}$ of the four solutions, near which one can find that $\tau_{v}(T)$ changes from Arrhenius dependence to a stronger temperature dependence on crossing $T_{c}$ practically the same as $T_{g}$. The length of the arrow, representing $\log \left[\tau_{\alpha}\left(T_{g}\right) / \tau_{v}\left(T_{g}\right)\right]$, is seen to increase with decreasing $C_{w}$. This trend is due to increasing intermolecular coupling of glucose solution on decreasing $C_{w}$, and again, it is consistent with the increase in $\tau_{\alpha}\left(T_{g}\right) / \tau_{v}\left(T_{g}\right)$ expected from the CM equation (2) by the corresponding increase in the coupling parameter $n$.

We have interpreted the $v$-relaxation as secondary relaxation contributed by the water component in aqueous solutions, and it is connected with the $\alpha$-relaxation in properties. The experimental evidence supporting this interpretation comes from the change in the temperature dependence of its dielectric strength $\Delta \varepsilon_{v}(T)$ at $T_{g}$ determined by $\tau_{\alpha}(T)$, and also, the rapid increase in $\Delta \varepsilon_{v}$ with an increase in $C_{w}$, as shown in Fig. 5 by the data of 20,26, and 30 wt. \% water solutions. In Fig. 6, the data of $\tau_{\alpha}(T)$ and $\tau_{v}(T)$ in water solutions of sucrose with $C_{w}=15,20,25$, and 30 , wt. $\%$ of water are presented. Dielectric spectra of these systems can be found in Figs. S9-S11 of the supplementary material. Again, $\tau_{v}(T)$ is much longer than $\tau_{\text {conf }}(T) \approx \tau_{\beta, \text { bulk }}(T)$. Also found are (1) the change in $T$-dependence of $\tau_{v}(T)$ at $T_{c} \approx T_{g}$, (2) the increase in $\log \left[\tau_{\alpha}\left(T_{g}\right) / \tau_{v}\left(T_{g}\right)\right]$ with decreasing $C_{w}$, (3) the change in $T$-dependence of $\Delta \varepsilon_{v}(T)$ at $T_{g}$ (see Fig. 7), and (4) the increase in $\Delta \varepsilon_{v}(T)$ with $C_{w}$ (see Fig. 7). These properties of $\tau_{v}(T)$ are similar to the glucose water solutions, 


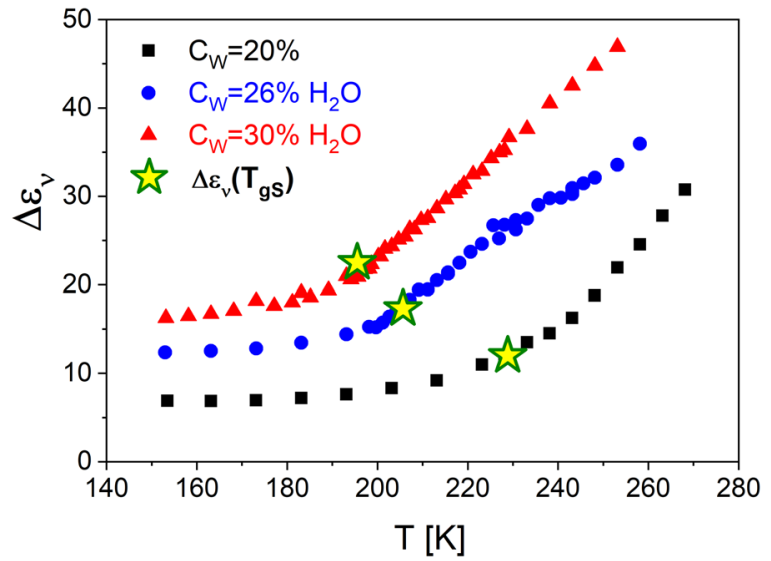

FIG. 5. Dielectric strength of the $v$-relaxation in water solutions of glucose with $C_{w}$ $=15,26$, and $30 \mathrm{wt}$. $\%$ of water plotted vs temperature. The yellow stars indicate dielectric strength $\Delta \varepsilon_{v}(T)$ at $T_{g s}$, as determined in Fig. 4.

but the effects in sucrose are larger due to higher $T_{g}$ of sucrose than glucose. This can be seen by the larger $\log \left[\tau_{\alpha}\left(T_{g}\right) / \tau_{v}\left(T_{g}\right)\right]$ of the sucrose solution than the glucose solution, both at $15 \mathrm{wt} \%$ of water.

Effects (1)-(4) were found generally in the other water solutions of deoxyribose, trehalose, fructose, and glycogen we studied. It is impracticable to present the collection of data in the paper. Some dielectric spectra of these systems are displayed in Figs. S12S14, S18, and S19 of the supplementary material. Nevertheless, as a representative of all the properties, we summarize the results of property (1) found in these hydrophilic solutes together with the

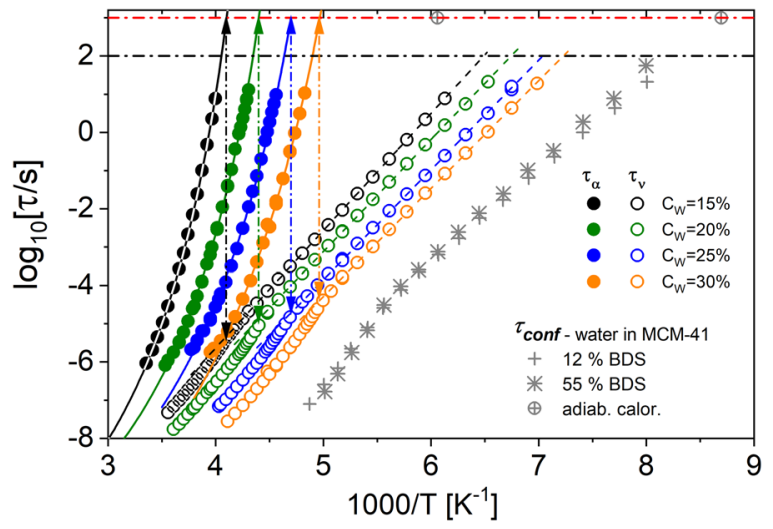

FIG. 6. Relaxation map: $\tau_{\alpha}(T)$ (solid circles) and $\tau_{v}(T)$ (open circles) in water solutions of sucrose with $C_{w}=15,20,25$, and 30 , wt. $\%$ of water plotted as a function of reciprocal temperature. Added for comparison are the dielectric data of $\tau_{\text {conf }}(T)$ in MCM- 41 with $12 \%$ (gray crosses) and 55 wt. \% (gray asterisks) of water $^{52}$ together with adiabatic calorimetry data (gray circled cross) of water confined within nanopores of MCM-41. ${ }^{56}$ Vertical arrows locate $1000 / T_{g} s$, where $T_{g} s$ is the glass transition of the solution. Continuous lines are VFT fits to $\tau_{\alpha}(T)$, and dashed lines are Arrhenius law fits to $\tau_{v}(T)$ below $T_{\mathrm{gS}}$

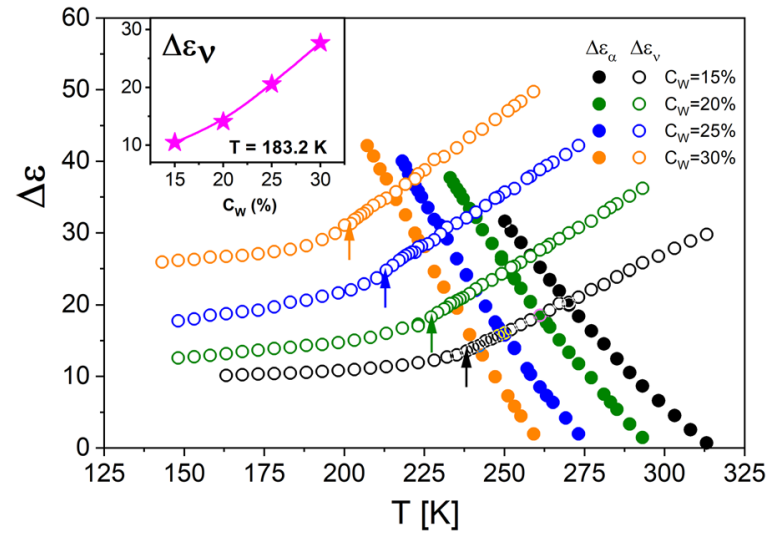

FIG. 7. Dielectric strength for $\alpha$ - and $v$-relaxation in water solutions of sucrose with weight water fraction $C_{w}=15,20,25$, and 30 , wt. \% plotted vs temperature. Vertical arrows indicate dielectric strength $\Delta \varepsilon_{v}(T)$ at $T_{g S}$, as determined in Fig. 6 . The inset shows, at a selected temperature $T_{x}=183.2 \mathrm{~K}$, the increase in $\Delta \varepsilon_{v}\left(T_{x}\right)$ with $C_{w}$.

oligomers of EG and propylene glycol ${ }^{23,64-68}$ in Fig. 8. Dielectric spectra of water mixtures with propylene glycol oligomers can be found in Figs. S15-S17 of the supplementary material. The temperature $T_{c}$ of the crossover of $\tau_{v}(T)$ from Arrhenius behavior well fitting the deep glassy state to stronger temperature dependence is plotted vs $T_{g S}$, the glass transition of the aqueous solutions, determined when $\tau_{\alpha}\left(T_{g S}\right)=10^{3} \mathrm{~s}$. The correlation is striking, confirming that property (1) holds for all the aqueous mixtures considered.

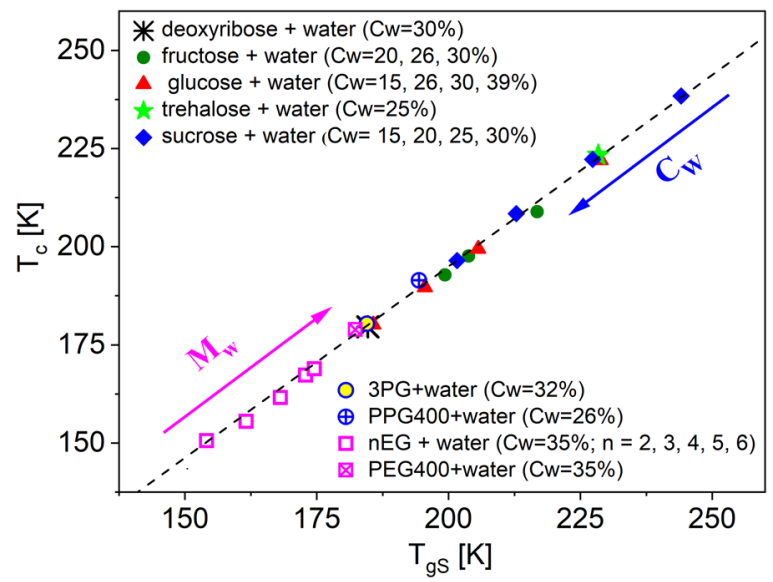

FIG. 8. Correlation plot of the temperature $T_{c}$ of the crossover of $\tau_{v}(T)$ from Arrhenius behavior vs $T_{g s}$, the glass transition of the aqueous solution, determined when $\tau_{\alpha}\left(T_{g S}\right)=10^{3} \mathrm{~s}$. The dashed line is a linear fit with coefficient 0.975 and zero offset. Data of glucose and sucrose are from Figs. 4 and 6, respectively. Trehalose, fructose, and deoxyribose data are from Refs. 23, 47, and 67. Data of aqueous mixtures of oligomers of EG are from Refs. 23 and $64-66$. Data of aqueous mixtures of oligomers of propylene glycol are from Ref. 68. 
For all figures presented in this section, $\tau_{v}(T)$ of water solutions of larger or more complex solutes is much longer than $\tau_{\beta, \text { bulk }}(T)$ of water. It is natural to ask if we can make $\tau_{v}(T)$ to get closer to $\tau_{J G, b u l k}(T)$ or even to become practically the same as $\tau_{\beta, b u l k}(T)$ by increasing $C_{w}$ in some aqueous solutions. The answer is in the positive as shown in Fig. 9 of the data of $\tau_{v}(T)$ of water solutions of several hydrophilic polymers. The examples given are $\mathrm{C}_{\mathrm{W}}=50 \mathrm{wt}$. $\%$ water mixture with PVME, ${ }^{69} 50$ wt. \% water with PVP, ${ }^{70,71} 59$ wt. \% of water with propylene glycol, ${ }^{72}$ and 38.6 wt. \% and 50 wt. \% of water with PHEMA, all with high molar $\%$ of water. ${ }^{73,74}$ The $\tau_{v}(T)$ of these aqueous polymer solutions are approaching $\tau_{\text {conf }}(T)$ of water confined in MCM-41, which are effectively $\tau_{\beta, b u l k}(T)$ of water.

Interestingly, despite the large molar fraction of water, $\tau_{v}(T)$ do not become shorter than $\tau_{\text {conf }}(T) \approx \tau_{\beta, b u l k}(T)$. This property (5) of $\tau_{v}(T)$ was first suggested by Fig. 4 of Ref. 14, where the $\tau_{\text {conf }}(T)$ in $20 \%$ of water nanoconfined in graphite oxide ${ }^{30}$ shorter than $\tau_{\text {conf }}(T)$ of water confined in MCM-41 was included. Since this figure is the origin of property (5) and it had inspired others, ${ }^{31,33}$ it is reproduced in the supplementary material. It turns out to be general for solutes having higher $T_{g}$ than that of ASW. It holds also in hydrated proteins and biomolecules, provided the level of hydration is sufficiently high, which will be demonstrated in Sec. V. By contrast, the $\tau_{v}(T)$ of the 35 wt. \% water solution of PEG600, shown also in Fig. 9, are much longer than $\tau_{\text {conf }}(T) \approx \tau_{\beta, \text { bulk }}(T)$. Dielectric spectra of $35 \mathrm{wt}$. $\%$ water solution of PEG600 are shown in Fig. S4 of the supplementary material. It is worthwhile to point out that there is a change in $\tau_{\text {conf }}(T)$ from the Arrhenius $T$-dependence at lower temperatures to a slightly stronger $T$-dependence on crossing some $T_{c}$, the value of

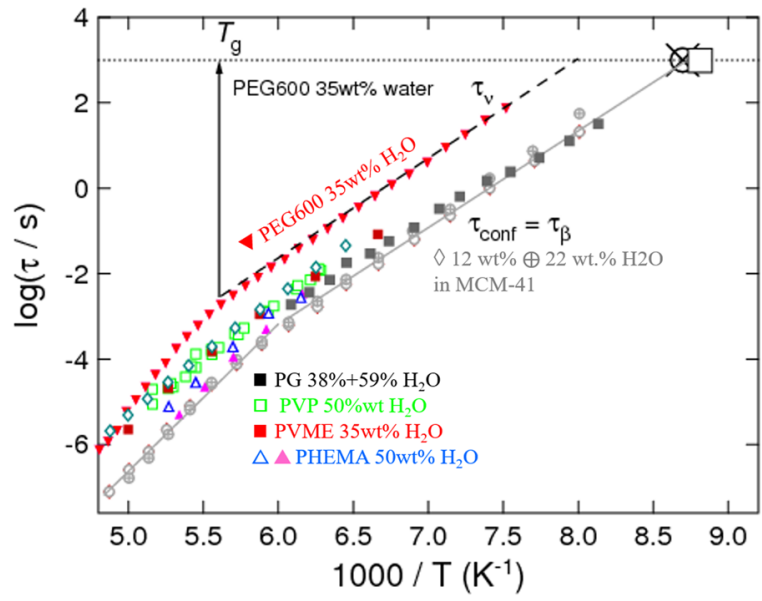

FIG. 9. $\log _{10} \tau_{v}(T)$ vs $1000 / T$ plots of the $v$-relaxation of the water component in various aqueous mixtures: 35 wt. \% water mixture with PEG600 (brown closed inverted triangles); 50 wt. \% water mixture with PVME (red closed squares); 50 wt. \% water with PVP (green open squares); 59 wt. \% PG; 38.6 wt. \%, 59 wt. $\%$ water with propylene glycol (black closed squares), and $50 \mathrm{wt} . \%$ of water with PHEMA (blue open triangles and magenta closed triangles, respectively). Shown by gray symbols are $\tau_{\text {conf }}(T)$ of water confined in molecular sieves MCM- 41 with pore diameter $2.14 \mathrm{~nm}$ at hydration levels $12 \mathrm{wt} . \%$ and $22 \mathrm{wt} . \%,{ }^{52}$ by adiabatic calorimetry of water confined within nanopores of MCM-41 (large black circled cross).

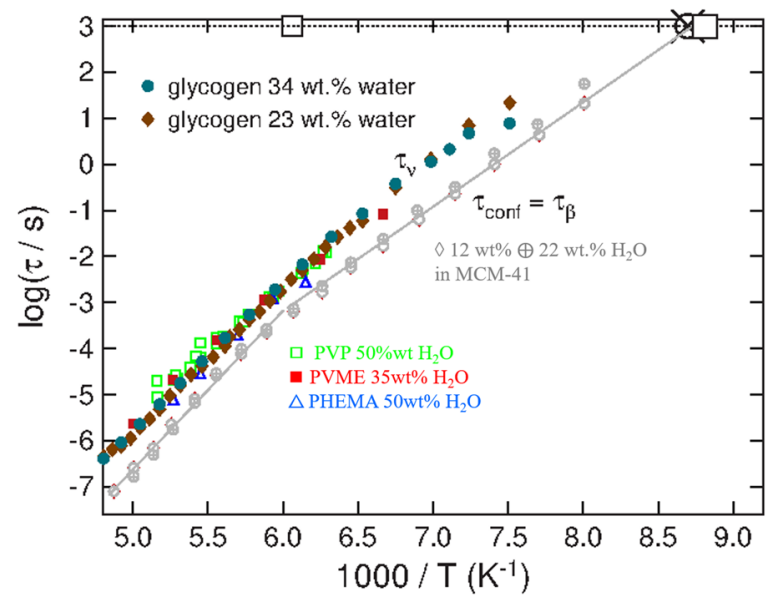

FIG. 10. Plots of $\log _{10} \tau_{v}(T)$ vs $1000 / T$ of the $v$-relaxation of the water component in water solutions of glycogen at 23 and $34 \mathrm{wt}$. \% water. Shown by gray symbols are $\tau_{\text {conf }}(T)$ of water confined in molecular sieves MCM-41 with pore diameter $2.14 \mathrm{~nm}$ at hydration levels $12 \mathrm{wt}$. \% and $22 \mathrm{wt}$. \%, by adiabatic calorimetry of water confined within nanopores of MCM-41 (large black circled cross) and water confined within silica pores (two large squares).

which increases with decreasing size of the confinement. ${ }^{30}$ The cause of the change is probably due to interaction of water with the confining surface. Therefore, the interaction at the surface is expected to be stronger if the size of the confinement is smaller, and correspondingly $T_{c}$ is higher. The slight change in $T$-dependence of $\tau_{\text {conf }}(T)$ does not change that $\tau_{\text {conf }}(T)$ continue to be approximately $\tau_{\beta, \text { bulk }}(T)$.

Currently, we are studying also the water solutions of glycogen, composed of highly branched linked glucopyranoside with high molecular weight $\left(\mathrm{M}_{\mathrm{w}}=270 \mathrm{kDa}\right)$. At $C_{w}=23 \%$ and $34 \%$, already there are about 2.7 and $4.8 \mathrm{H}_{2} \mathrm{O}$ per saccharide ring monomers, respectively. Although ice is also present for $C_{w}=34 \%$, most of the water is in the amorphous state at lower temperatures, thanks to the highly branched structure of the polysaccharide that confined the water in excess to nanometric interstices: the $v$-relaxation present is detected and characterized. Here, we show in Fig. 10 the temperature dependences of relaxation times of $\tau_{v}(T)$ of glycogen with $C_{w}=23 \%$ and $34 \%$. It can be seen by inspection that $\tau_{v}(T)$ are close to $\tau_{\text {conf }}(T) \approx \tau_{\beta, \text { bulk }}(T)$ but never becomes shorter than the latter, despite the large molar fraction of water in the solution of glycogen. The same behavior of $\tau_{v}(T)$ of glycogen is found for amorphous water at even higher $C_{w}=34 \%, 54 \%$, and 77 wt. \% of water (not included in Fig. 10). The complete set of experimental data of our study of water solutions of glycogen will be published elsewhere.

\section{THE $v$-RELAXATION IN HYDRATED BIOMOLECULES}

Five general properties of the $v$-relaxation have been brought out by experimental studies of aqueous solutions of hydrophilic molecules and macromolecules. Proteins and biomolecules have more complex structures, and their physical relation to water can be different from homogeneous water solutions of molecules. 
For example, water added to globular proteins forms a hydration shell and the association of water with different parts of the protein depends on the hydration level. Moreover, water has a peculiar role in promoting dynamics in hydration shells and interlaying between the hydrogen bonds governing the structure of proteins. A recent study $^{75}$ on series of oligo- and poly-peptides at different hydration levels reports a scenario quite different from those reported in Figs. 3, 4, and 6, with a lesser importance played by the molecular weight of the solute with respect to that of water, that appears to have a main role to "slave" the dynamics of the peptides. Therefore, it is not clear if the $v$-relaxation shows up in hydrated proteins and biomolecules and if present that it possesses all the properties found in homogeneous water solutions. Our studies ${ }^{14,23-25,41-44,76}$ as well as others ${ }^{33,77-79}$ in the past had observed properties (1)-(4) in some hydrated proteins. We have interpreted the observed fast relaxation as the $v$-relaxation from water coupled to the protein in the hydration shell. It has properties (1)-(4), and it is responsible for the "dynamic transition" detected by Mössbauer and neutron scattering spectroscopy occurring at temperature $T_{d}$, which depends on the time scale of the instrument used ${ }^{37,80}$ or neutron scattering. ${ }^{38-40,81}$ On the other hand, interested mainly on the dynamic transition, others have paid little attention to the $v$-relaxation, although there are notable exceptions. ${ }^{33,34,46,77-79,82-86}$

While properties (1)-(4), as defined in Sec. IV, are more widely known to apply, the general validity of property (5) of hydrated proteins and biomolecules is not clear. As a reminder, property (5) implies that $\tau_{v}(T)$ of all hydrated proteins and biopolymers can approach $\tau_{\beta, \text { bulk }}(T)$ of water on increasing the hydration level, but $\tau_{v}(T)$ cannot be shorter than $\tau_{\beta, \text { bulk }}(T)$. It was examined for data taken by neutron scattering over the short time range in bovine serum albumin (BSA) for the first time in Ref. 77 and followed only by the authors of Ref. 30 also in BSA with improved experimental data. For this reason and the important implication of property (5) on experiments, we focus our attention on examining the validity of property (5) in hydrated proteins and biomolecules in the remainder of this paper. This study confers a bonus of showing clearly the mistake made in identifying the $v$-relaxation observed a short time by neutron scattering with the $\alpha$-relaxation in hydrated myoglobin ${ }^{38}$ to be discussed next.

\section{A. Hydrated myoglobin}

We start with the hydrated myoglobin and the data collected by Doster ${ }^{38,39,87}$ and by others. ${ }^{26,78}$ Shown in Fig. 11 are the relaxation times of myoglobin hydration water (HW) from neutron scattering with resolution time $\sim 10^{-9} \mathrm{~s},{ }^{88} \mathrm{NMR},{ }^{79}$ Mössbauer spectroscopy, ${ }^{88,89}$ iso-frequency specific heat spectroscopy ${ }^{38}$ of hydrated myoglobin $(h=0.4 \mathrm{~g} / \mathrm{g})$, and the dielectric relaxation $(h=0.33) .{ }^{26,78}$ Both the $\alpha$-relaxation and the $v$-relaxation were detected in the dielectric experiment, and their relaxation times, $\tau_{\alpha}(T)$ and $\tau_{v}(T)$, are shown by red open and large closed squares, respectively, in Fig. 11. There is approximate agreement between the dielectric (open red squares) and the calorimetric (open black diamonds) $\tau_{\alpha}(T)$. It is important to observe that the dielectric $\tau_{v}(T)$ overlap with the relaxation times from neutron scattering, NMR, and Mössbauer spectroscopy, which indicates that the latter are also $\tau_{v}(T)$ and should not be confused with the much longer $\tau_{\alpha}(T)$. The mistake was made by others ${ }^{38,39}$ in associating all of the relaxation times

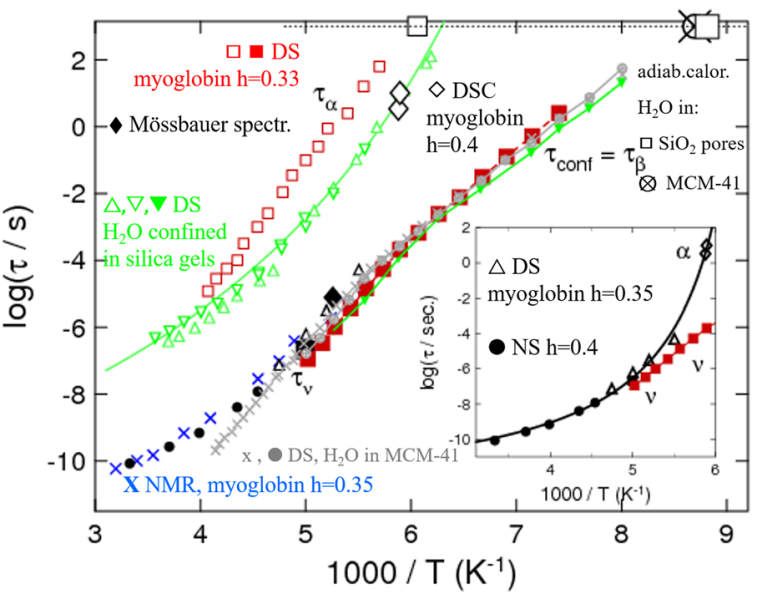

FIG. 11. Collection of hydrated myoglobin data. The black closed circles are from neutron scattering, open black triangles are dielectric relaxation data adopted by Doster in Fig. 3 in Ref. 38, the lone large closed square is from Mössbauer spectroscopy, and the two open diamonds at the top are from specific heat spectroscopy all taken from Fig. 3 of Ref. 38 . The fit to a VFT-equation provided in Fig. 3 of Ref. 38 is not shown. The same symbols as in Fig. 3 of this reference are used to represent the relaxation time data of HW. These same data appear also in the main part of the figure. Added are the dielectric $\tau_{v}$ and $\tau_{\alpha}$ of hydrated myoglobin (red closed and open squares, respectively). The NMR relaxation times of hydrated myoglobin at $h=0.35$ are represented by the multiplication signs. Shown by gray symbols are $\tau_{\text {conf }}(T)$ of water confined in molecular sieves MCM-41 with pore diameter $2.14 \mathrm{~nm}$ at hydration levels $12 \mathrm{wt}$. $\%$ and $22 \mathrm{wt}$. \%, by adiabatic calorimetry of water confined within nanopores of MCM-41 (large black circled cross) and water confined within silica pores (two large squares). The green closed and open triangles are the dielectric $\tau_{\text {conf }}(T)$ and the associated $\tau_{\alpha, \text { conf }}(T)$ of water confined in pores of silica gels. The inset is basically a reproduction of Fig. 3 of Doster in Ref. 38, which is the Arrhenius plot of data called the $\alpha$-relaxation rate $k_{S} \equiv 1 / \tau_{\alpha}$ for hydrated myoglobin $(\mathrm{HW})$ at $h=0.4$ with part of the dielectric data taken from Ref. 26. The line is the VFT fit by Doster ${ }^{38}$ connecting the $\tau_{\alpha}$ from calorimetry and $\tau_{v}$ from dielectric, NMR, and neutron scattering, which is meaningless.

from neutron scattering, NMR, and Mössbauer spectroscopy, and the high temperature part of the dielectric $\tau_{v}(T)$ from Ref. 26, with the structural $\alpha$-relaxation times $\tau_{\alpha}$, which are distinctly different (see Fig. 11). This was done in Fig. 3 of Ref. 38 for HW by Doster and reproduced here in the inset of Fig. 11 by the solid line, which is the fit by the Vogel-Fulcher-Tammann (VFT) equation given in Ref. 38. After rectifying this mistake, it is clear that the dynamic transition is due to the $v$-relaxation entering the time scales of the Mössbauer spectroscopy and the neutron scattering instrument but not $\alpha$-relaxation as claimed by others. ${ }^{38,87}$

Included in Fig. 11 are the $\tau_{\text {conf }}(T)$ of water nanoconfined obtained by dielectric relaxation (gray symbols) ${ }^{52}$ and by adiabatic calorimetry (located at $\left.10^{3} \mathrm{~s}\right)^{56}$ presented before in Figs. 9 and 10, and it is supposed to be approximately $\tau_{\beta, b u l k}(T)$ of water when it has the Arrhenius $T$-dependence and $\tau_{v}(T)$ of the $v$-relaxation after crossing $T_{c}$ of the nanoconfined water. In addition, both the $\tau_{\text {conf }}(T)$ and $\tau_{\alpha}(T)$ of water confined in silica gels ${ }^{55}$ are shown by green symbols.

By comparing the $\tau_{v}(T)$ of hydrated myoglobin from all spectroscopies with $\tau_{\text {conf }}(T)$ of water in Fig. 11, it is clear that they 
are practically the same. Also, $\tau_{v}(T)$ does not become shorter than $\tau_{\beta, b u l k}(T)$. Thus, the important conclusion drawn is that property (5) holds in hydrated myoglobin at the optimal hydration level of $h=0.33-0.40$. We proceed to examine other hydrated proteins and biomolecules to see if property (5) also holds provided the level of hydration is sufficiently high.

\section{B. Hydrated lysozyme}

The presence of $\alpha$-relaxation much slower than the $v$-relaxation seen in hydrated myoglobin at $h=0.34-0.40$ is confirmed in hydrated lysozyme by the dielectric measurements by Nakanishi and Sokolov. ${ }^{46}$ Three processes were found, but we consider only the slowest and the fastest, which are the $\alpha$-relaxation and the $v$ relaxation, respectively. The relaxation times $\tau_{\alpha}(T)$ and $\tau_{\nu}(T)$ are plotted in Fig. 12, where included are dielectric $\tau_{v}(T)$ of hydrated lysozyme at hydration level $h \sim 0.4$ from Ref. 90 and also $\tau_{v}(T)$, the neutron scattering relaxation times for nonexchangeable protons in the protein molecules. ${ }^{91}$ The wide separation in time scale between $\tau_{\alpha}(T)$ and $\tau_{v}(T)$ in Fig. 12 for all temperatures makes

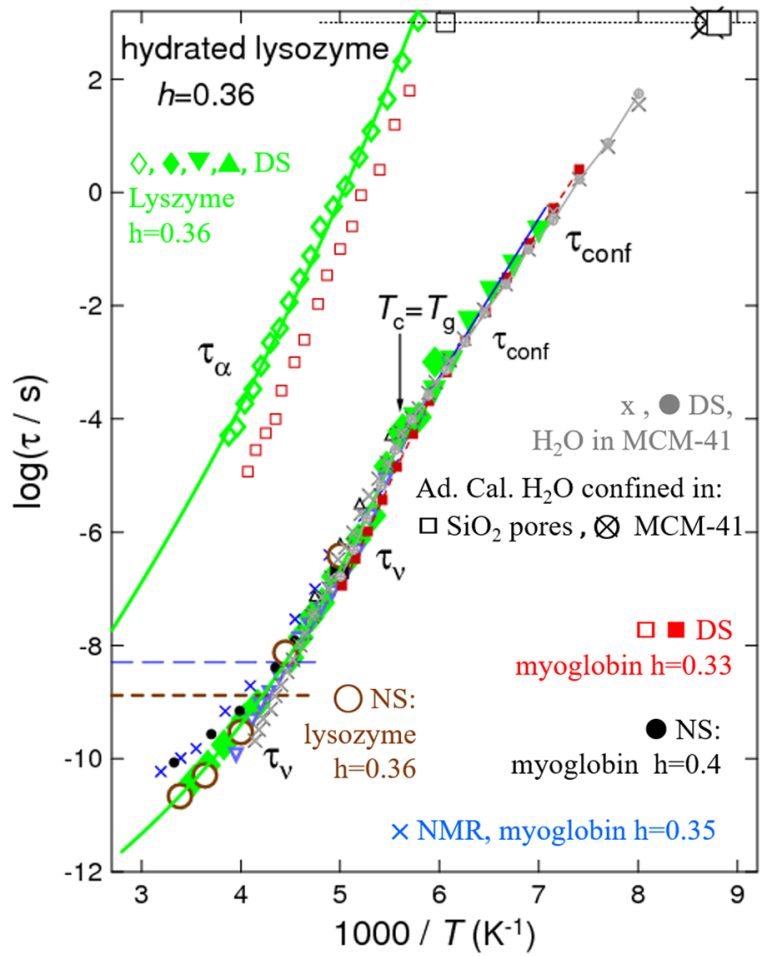

FIG. 12. The green open and closed diamonds stand for dielectric relaxation times of $\tau_{\alpha}$ and $\tau_{v}$ of hydrated lysozyme at $h=0.36$. The green inverted triangles are dielectric $\tau_{v}$ from a different source. The large brown open circles present the neutron scattering relaxation times for nonexchangeable protons in the protein molecules. The upper and lower dashed horizontal lines represents the value of $\tau_{v}$ satisfying the condition $\tau_{\text {res }}=0.2 \tau_{v}$ and $\tau_{\text {res }}=\tau_{v}$, respectively, for the HFBS spectrometer used. The gray symbols are dielectric $\tau_{\text {conf }}(T)$ as in Fig. 11. Included are the data of hydrated myoglobin shown before in Fig. 11. Dielectric $\tau_{v}$ and $\tau_{\alpha}$ (red closed and open squares, respectively), NMR relaxation times (multiplication signs), neutron scattering (black closed circles), dielectric relaxation data adopted by Doster (open black triangles). clear that the process probed by Mössbauer spectroscopy neutron scattering at short time scale is the $v$-relaxation and not the a-relaxation.

All the data of hydrated myoglobin in Fig. 11 are included for comparison. Remarkably, the dielectric and neutron scattering $\tau_{v}(T)$ of the hydrated lysozyme follow closely those of the hydrated myoglobin over the entire broad temperature range. The glass transition temperatures of the two hydrated globular proteins are approximately the same as indicated by nearly the same $\tau_{\alpha}(T)$ and nearly the same $T_{c}$ at which $\tau_{v}(T)$ changes its temperature dependence. In particular, there is also good correspondence between $\tau_{v}(T)$ and $\tau_{\text {conf }}(T) \approx \tau_{\beta, \text { bulk }}(T)$ of water nanoconfined obtained by dielectric relaxation (gray symbols), and again $\tau_{v}(T)$ does not become shorter than $\tau_{\text {conf }}(T) \approx \tau_{\beta, \text { bulk }}(T)$. Hence, the hydrated lysozyme has property (5).

\section{Hydrated bovine serum albumin (BSA)}

Kyritsis and co-workers ${ }^{33}$ studied mixtures of water and another globular protein, bovine serum albumin (BSA), over wide ranges of composition from 0.2 to $40 \mathrm{wt}$. $\%$ water by DSC and dielectric relaxation spectroscopy. The glass transition was detected by DSC. The relaxation times $\tau_{\alpha}(T)$ and $\tau_{v}(T)$ of the $\alpha$-relaxation and $\nu$-relaxation in hydrated BSA with $28 \%$ and $40 \%$ water obtained by dielectric measurements are replotted in Fig. 13. For comparison, we include all the $\tau_{\alpha}(T)$ and $\tau_{v}(T)$ data of hydrated myoglobin and $\tau_{\text {conf }}(T)$ of nanoconfined water in Fig. 11.

At all temperatures, $\tau_{\alpha}(T)$ is many orders of magnitude longer than $\tau_{v}(T)$, and undoubtedly, the dynamic transition observed in hydrated BSA occurs when $\tau_{v}(T)$ enters into the time resolution of the instrument. Although $\tau_{v}(T)$ is not nearly equal to $\tau_{\text {conf }}(T)$ as found in hydrated myoglobin and lysozyme, the differences between the two are typically less than half a decade, with $\tau_{v}(T)$ slower than $\tau_{\text {conf }}(T)$. A further increase in water content up to $80 \%{ }^{76}$ does not

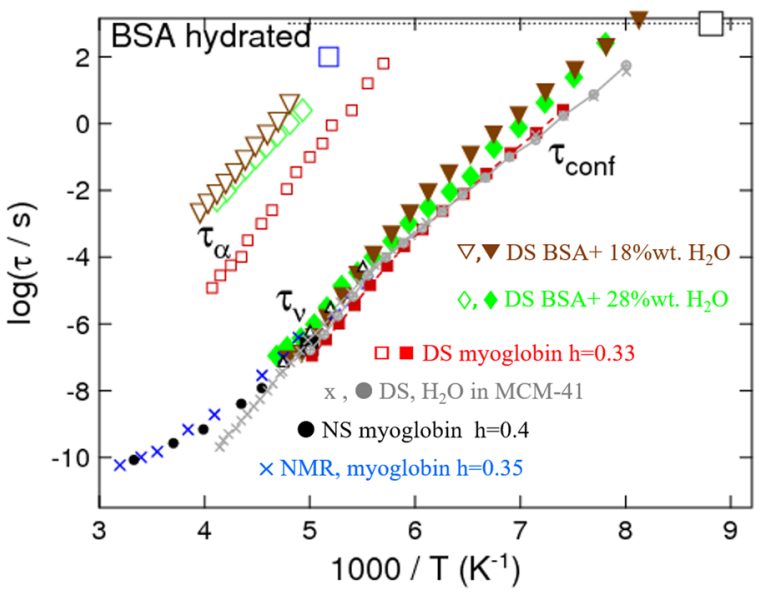

FIG. 13. Temperature dependence of the relaxation times for the a-relaxation (open symbols) and the $v$ relaxation (closed symbols) of the hydrated BSA for samples of two different water fractions of $18 \%$ (brown inverted triangles) and $28 \%$ (green diamonds). The glass transition temperature is located by the blue square. Added for comparison are the data of hydrated myoglobin and $\tau_{\text {conf }}(T)$ shown before in Fig. 11. 
make $\tau_{v}(T)$ shorter than $\tau_{\text {conf }}(T) \approx \tau_{\beta, b u l k}(T)$. Hence, property $(5)$ is verified in hydrated BSA.

\section{Hydrated RNase A}

We have studied by dielectric spectroscopy the dynamics of the globular protein ribonuclease A (RNase A) at hydration level $h=0.4$. Similar to what usually occurs in dielectric spectroscopy studies on hydrated biomolecules, ${ }^{33,46}$ the low frequency part of loss spectra at high temperature is dominated by a strong dc conductivity contribution $\sigma_{\mathrm{dc}} /\left(\varepsilon_{0} \omega\right)$. Taking advantage from the fact that complex permittivity has been measured, we calculated a quantity that approximates the dielectric loss spectra, shown in Fig. 14, that is proportional to the logarithmic derivative of the real part of permittivity, following the procedure illustrated in Ref. 92, that helps gain information on the low frequency part of losses minimizing the conductivity contribution. Spectra display the same features shown by hydrated lysozyme: ${ }^{46}$ three processes are present, two slow processes (that we label as I- and II-) and a faster process that has the features of the $v$-relaxation described before. In particular, the evolution with temperature of the relative amplitude of $v$-relaxation follows an increasing behavior similar to that shown by $v$-relaxation in aqueous mixtures of saccharides (see Figs. 5 and 7). At very low temperatures, loss spectra show a featureless power law behavior of the kind of $\varepsilon^{\prime \prime} \sim \omega^{-c}$ with $c \sim 0.1$, the nearly constant law phenomenon, result of caged dynamics, already observed in proteins.

In Fig. 15(a), we show the relaxation times $\tau_{s I}(T), \tau_{s I I}(T)$, and $\tau_{v}(T)$. The slow processes have a Vogel-Fulcher-Tammann temperature dependence, whereas $\tau_{v}(T)$ has an Arrhenius behavior at a low temperature (with activation energy around $49 \mathrm{KJ} / \mathrm{mol}$ ), but it

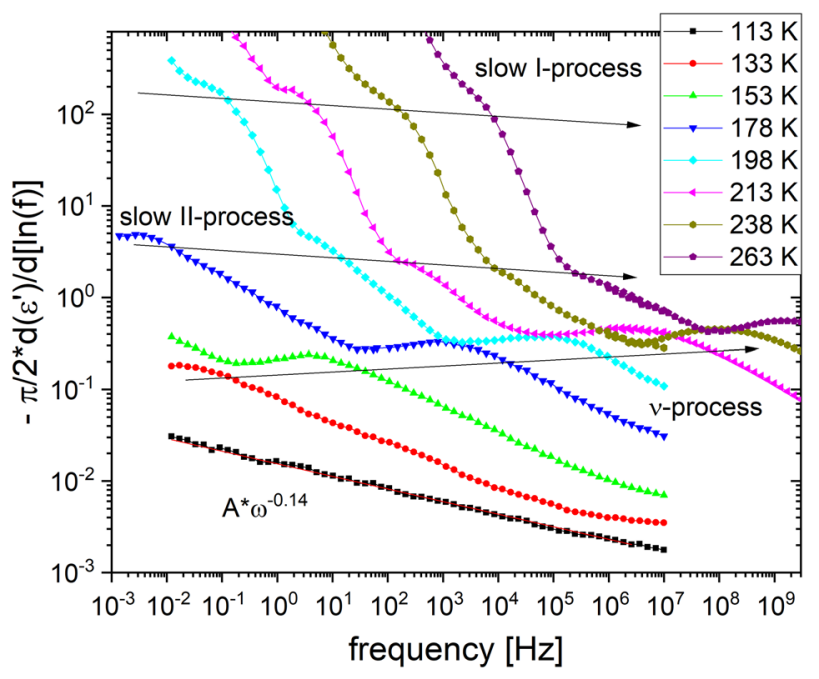

FIG. 14. Loss spectra of hydrated RNase $A$ at $h=0.4$ at selected temperatures, as obtained by the derivative of the real part of permittivity. The power law fit to the spectrum at $T=113 \mathrm{~K}$ has a bilogarithmic slope of -0.14 , characteristic of a nearly constant loss. Arrows show the amplitude and frequency scale evolution with increasing temperature of slow I-process, II-process, and v-processes, from up to down, respectively.

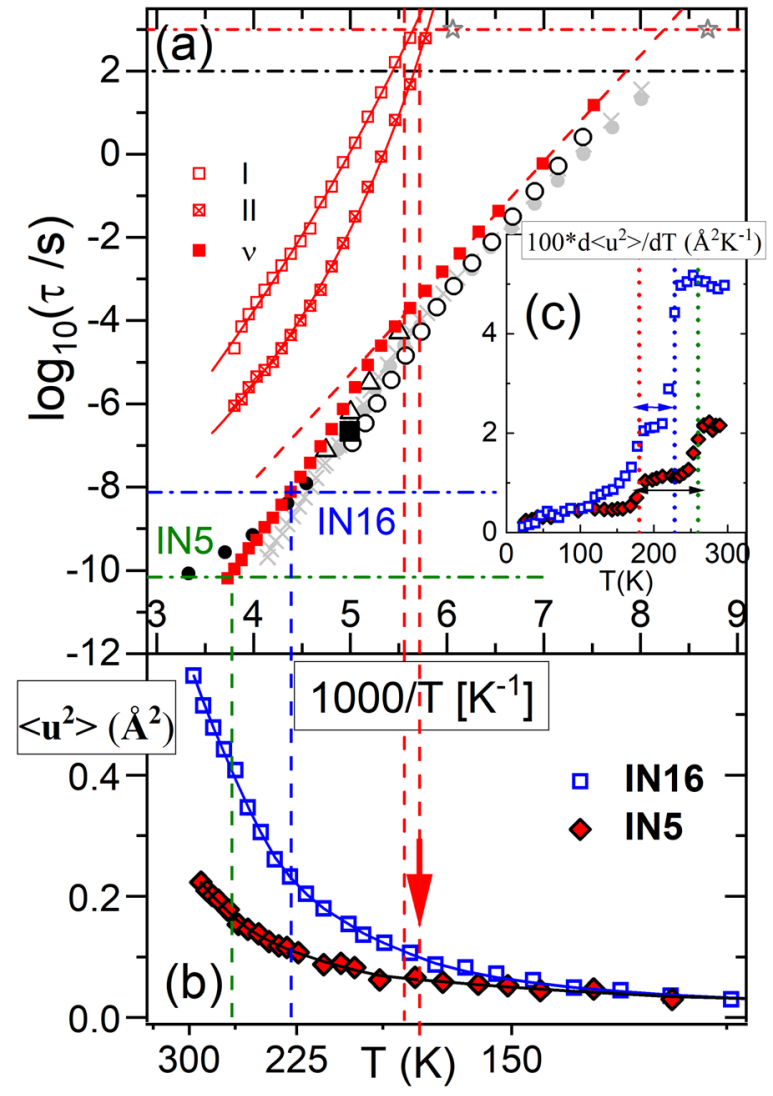

FIG. 15. (a) Temperature dependence of the relaxation times for the slow I-process (red open squares), II-process (red crossed squares), and the $v$ relaxation (red closed squares) of the hydrated RNAse $h=0.4$. Continuous and dashed lines are fits with VFT and Arrhenius functions. Added for comparison are the data of $\tau_{\text {conf }}(T)$ in MCM shown before in Fig. 11 (gray symbols, same as before) and those of hydrated myoglobin (black symbols), same as in Fig. 11 with added dielectric data (open black circles) taken from Ref. 26. Black, red, blue, and green horizontal dashed-dotted lines indicate, respectively, $1 \mathrm{ks}, 100 \mathrm{~s}$, and the time scale where the relaxation enter into the frequency window of the IN 16 and IN 5 spectrometers (see text). (b) Mean squared displacements $\left\langle u^{2}\right\rangle$ of hydrated RNase ${ }^{90}$ as obtained by neutron scattering using spectrometers IN16 and IN5 plotted vs reciprocal temperature scale [same scale as in panel (a)]. Lines are spline curves used to determine the local slope. Green vertical dashed, blue, and red lines indicate the dynamic transition seen by IN5, IN16, and the glass transition temperature. (c) Local derivative of $\left\langle u^{2}\right\rangle$ with respect to $T$ obtained by numerical analysis of the data in panel $(b)^{43}$ plotted vs temperature.

shows a change of slope on crossing the temperature range of 175$180 \mathrm{~K}$. Around the same temperature, both slow processes attain a relaxation time of the order of $1 \mathrm{ks}$. The glass transition temperature is probably near this crossover temperature, as it can also be inferred on the base of a structural change shown by Debye-Waller factors, obtained by X-ray diffraction. ${ }^{94}$ As a comparison, the data of hydrated myoglobin (black symbols) and confined water $\tau_{\text {conf }}(T)$ shown before in Fig. 11 (gray symbols) are also displayed. $\tau_{v}(T)$ of hydrated RNAse is approaching the latter but never passes that time scale. 
This system has also been studied by elastic incoherent neutron scattering. ${ }^{93}$ It is quite instructive to compare the data of mean squared displacement (MSD) by two different spectrometers IN5 and IN16, plotted in Fig. 15(b). It occurs that MSD has some changes in temperature behavior, visible in Fig. 15(b) around $265 \mathrm{~K}$ (for IN5), $228 \mathrm{~K}$ (for IN16), and $175 \mathrm{~K}$ (for both). Actually, the region between 175 and $180 \mathrm{~K}$, where MSD increases significantly from the low temperature values, corresponds in Fig. 15(a) to the region of glass transition. Both spectrometers register such an increase in MSD around the same temperature, irrespective of their time scale resolution. This is expected since it is the manifestation of the softening of the system on crossing the glass transition, and that is reflected by the change in temperature dependence of the caged dynamics responsible for the MSD at this time/temperature range. ${ }^{42}$ There are other two changes of the MSD corresponding to the higher dynamic transition temperature $T_{d}$ : horizontal green and blue dashed-dotted lines in panel (a) indicate the time scale where the relaxation enters into the frequency window of IN5 and IN16 spectrometers, for which the dynamic transition should occur, following the rule of thumb $\tau_{\text {res }} \sim 0.2 \tau_{v}{ }^{81}$ The temperatures $T_{d}$ where $\tau_{v}\left(T_{d}\right)$ crosses those lines are $265 \mathrm{~K}$ and $228 \mathrm{~K}$, compatible with the dynamic transition estimated by the change of slope of MSD of IN5 and In16, respectively [see Fig. 15(c) and Ref. 43]. It is once again demonstrated that the $v$-relaxation entering the time scales of the spectrometer is responsible for dynamic transition, not the $\alpha$-one, unless the two are not merged together already. Also, in this case, property (5) is fulfilled since $\tau_{v}(T)$ is approaching $\tau_{\text {conf }}(T)$ but is always longer.

\section{E. Hydrated elastin and collagen}

So far, the verification of property (5) is restricted to the hydrated globular proteins. We now consider the hydrated fibrous proteins, elastin, and collagen studied by several groups. Lusceac used ${ }^{2} \mathrm{H}$ and ${ }^{13} \mathrm{C}$ NMR techniques, ${ }^{79}$ while Gainaru et al. ${ }^{85}$ and Panagopoulou et al. ${ }^{34}$ used dielectric spectroscopy over a wide hydration range (0-23 wt. \%) and DSC for their studies. The dielectric relaxation time $\tau_{v}(T)$ of the $v$-relaxation determined over a wide range is reproduced in Fig. 16, where also shown are the $\tau_{\text {conf }}(T)$ of water nanoconfined in MCM-41 $1^{52,56}$ and silica gels. ${ }^{55,57}$ The data of Gainaru et al. are plotted but not those of Panagopoulou et al. to avoid overcrowding. Nevertheless, the data of Panagopoulou et al. of elastin with $h=0.23$ obtained over the range, $10^{-7}<\tau_{v}(T)<10 \mathrm{~s}$, are well described by the Arrhenius temperature dependence with a prefactor of $10^{-19.39} \mathrm{~s}$ and activation energy of $0.52 \mathrm{eV}$ or $50.2 \mathrm{~kJ} / \mathrm{mol}$ and represented by the thick green line in Fig. 16. This value of the activation energy is nearly the same as $48 \mathrm{~kJ} / \mathrm{mol}$ of $\tau_{\text {conf }}(T)$ of water nanoconfined in MCM-41 and silica gels or that of $\tau_{\beta}(T)$ of water (see the discussion in Sec. III A). This is unsurprising because $\tau_{v}(T)$ of elastin and collagen from the work of Gainaru et al. as well as $\tau_{v}(T)$ of elastin from the work of Panagopoulou et al. overlap $\tau_{\text {conf }}(T) \approx \tau_{\beta, \text { bulk }}(T)$ of water nanoconfined at temperatures below $T_{c}$. The change in temperature dependence of $\tau_{\text {conf }}(T)$ to a stronger $T$-dependence is due possibly to interaction of the water with the confinement interface. Notwithstanding, the change in temperature dependence is minor, indicating weak interaction. Had this interaction not present, the Arrhenius dependence of $\tau_{\text {conf }}(T) \approx \tau_{\beta, \text { bulk }}(T)$ will continue and represents that of $\tau_{\beta, \text { bulk }}(T)$ over the entire

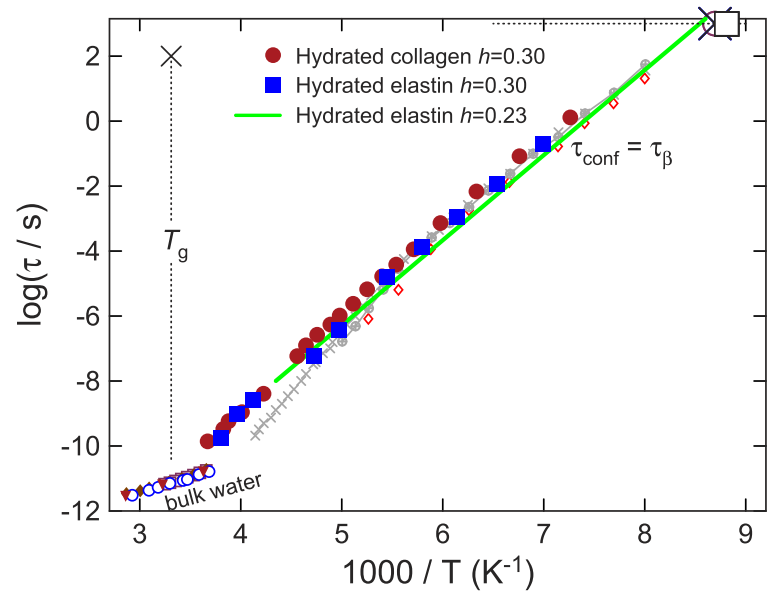

FIG. 16. Temperature dependence of the relaxation times for the $v$ relaxation of uncrystallized water for hydrated elastin and collagen at water fractions $h=0.30$ indicated in the plot. ${ }^{85}$ The thick green line represents the Arrhenius temperature dependence specified in the text, which approximates the data of elastin at $h=0.23{ }^{34}$ Added are the $\tau_{\text {conf }}(T)$ of water confined represented by red open diamonds, ${ }^{55}$ gray squares, and crosses, ${ }^{52}$ symbols on the top right corner from adiabatic calorimetry. ${ }^{56,57}$ Shown in the left lower corner are the neutron and high frequency dielectric relaxation data of bulk water, presented before in the inset of Fig. 2. The dotted line indicates the location of $1000 / T_{g}$ with $T_{g}=302 \mathrm{~K}$ of elastin at $h=0.224$ determined by DSC from Ref. 34 .

temperature range. Thus, not only property (5) is verified in elastin and collagen, but also the data of $\tau_{v}(T)$ provide $\tau_{\beta, b u l k}(T)$ over ten decades. Moreover, if extrapolated to higher temperature, $\tau_{v}(T)$ are not far from the neutron scattering and high frequency dielectric relaxation times of $\tau_{\alpha, b u l k}(T)$ shown in the left lower corner. At these high temperatures, cooperativity of the $\alpha$-relaxation is practically totally removed or $n=0$, and $\tau_{\alpha, b u l k}(T)$ is no different from $\tau_{\beta, b u l k}(T)$.

\section{CONCLUSIONS}

From the experimental data and the results from the analyses given, we make the following conclusions on the dynamics of bulk water, aqueous mixtures, and hydrated biomolecules.

For bulk water in the low and high temperature ranges where crystallization is averted, its structural $\alpha$-relaxation has narrow frequency dispersion or nearly exponential time correlation function. The temperature dependence of the a-relaxation times $\tau_{\alpha, b u l k}(T)$ is well described by a single Vogel-Fulcher-Tammann function, and there is no sign of the fragile-strong transition. The value of the fragility index $m$ is 44 . The low value of $m$ and large $\beta_{K}$ of water are in accord with the anticorrelation between the two quantities. ${ }^{95}$ The JG $\beta$-relaxation of bulk water exists, and its presence is brought out by the fast relaxation with Arrhenius temperature dependence observed in water confined in nanometer spaces. The JG $\beta$-relaxation times, $\tau_{\beta, b u l k}(T)$, are obtained by identifying with the relaxation times $\tau_{\text {conf }}(T)$ of water in nanoconfinement. The ratio $\tau_{\alpha, b u l k}\left(T_{g}\right) / \tau_{\beta, b u l k}\left(T_{g}\right)$ is smaller than most glass-formers, 
and it corresponds to the Kohlrausch a-correlation function, exp$\left(t / \tau_{\alpha, b u l k}\right)^{1-n}$, having the value of 0.90 .

The glass transition temperatures of most aqueous mixtures with hydrophilic molecules and polymers as well as hydrated proteins and biomolecules are higher than that of bulk water. Coexisting with the $\alpha$-relaxation is a secondary $v$-relaxation originating from the water component, as evidenced by the increase in the dielectric strength $\Delta \varepsilon_{v}(T)$ with $C_{w}$, the wt. $\%$ of water. The $v$-relaxation is strongly connected to the $\alpha$-relaxation in its properties, including the change in $T$-dependence of $\tau_{v}(T)$ and $\Delta \varepsilon_{v}(T)$ at $T_{c} \approx T_{g}$ and the decrease in $\log \left[\tau_{\alpha}\left(T_{g}\right) / \tau_{v}\left(T_{g}\right)\right]$ with increasing $C_{w}$. These connections are exactly as found in binary mixtures of van der Waals glass-formers, ${ }^{24}$ plus more to show the coupling between the two relaxations. Therefore, the $\nu$-relaxation is the JG $\beta$-relaxation of these systems. At lower water content, its relaxation time $\tau_{v}(T)$ is longer than $\tau_{\beta, b u l k}(T)$, but $\tau_{v}(T)$ decreases with increase in $C_{w}$, and $\tau_{v}(T)$ can become the same as $\tau_{\beta, b u l k}(T)$ at sufficiently high water content, provided crystallization does not intervene. However, $\tau_{v}(T)$ does not become shorter than $\tau_{\beta, b u l k}(T)$ at even higher water content. Thus, $\tau_{\beta, b u l k}(T)$ is the lower bound of $\tau_{v}(T)$ for all aqueous mixtures and hydrated biomolecules.

In connection with the so-called dynamic transition at $T_{d}$ observed by Mössbauer and neutron scattering spectroscopies, very often linked to the biological function of the proteins, ${ }^{96}$ it is important to note that it is triggered by the $v$-relaxation entering the time scale of the instruments and not the a-relaxation. That is, at $T_{d}$, we have either $\tau_{v}\left(T_{d}\right)$ or $\tau_{\beta, b u l k}\left(T_{d}\right)$, depending on the hydration level, matching the time scale of the instrument. Although present, the $\alpha$-relaxation is not involved because $\tau_{\alpha}\left(T_{d}\right)$ is many orders of magnitude longer than $\tau_{v}\left(T_{d}\right)$ or $\tau_{\beta, b u l k}\left(T_{d}\right)$.

\section{SUPPLEMENTARY MATERIAL}

See supplementary material for a comparison between relaxation times of $v$-relaxation in aqueous mixtures, and relaxation of nanoconfined water vs temperature is provided. The dielectric spectra of some systems studied in this paper are presented.

\section{ACKNOWLEDGMENTS}

We warmly thank Marco Bianucci for assistance in dielectric spectroscopy measurements. This work was supported in part by the National Basic Research Program of China (973 Program No. 2015CB856805), National Natural Science Foundation of China (NSFC) (Grant Nos. 51871193 and 11474247), and Key Research and Development Program of Hebei Province (Grant No. 18391502D).

\section{REFERENCES}

1'S. Capaccioli and K. L. Ngai, J. Chem. Phys. 135, 104504 (2011).

${ }^{2}$ G. P. Johari, A. Hallbrucker, and E. Mayer, Nature 330, 552 (1987).

${ }^{3}$ G. P. Johari, A. Hallbrucker, and E. Mayer, J. Chem. Phys. 95, 2955 (1991).

${ }^{4}$ K. Winkel, D. T. Bowron, T. Loerting, E. Mayer, and J. L. Finney, J. Chem. Phys. 130, 204502 (2009).

${ }^{5}$ K. Winkel, M. S. Elsaesser, E. Mayer, and T. Loerting, J. Chem. Phys. 128, 0445101 (2008).

${ }^{6}$ K. Winkel, M. S. Elsaesser, M. Seidl, M. Bauer, E. Mayer, and T. Loerting, J. Phys.: Condens. Matter 20, 494212 (2008).
${ }^{7}$ C. A. Angell, Chem. Rev. 102, 2627-2650 (2002).

${ }^{8}$ Y. Z. Yue and C. A. Angell, Nature 427, 717 (2004).

${ }^{9}$ A. Minoguchi, R. Richert, and C. A. Angell, Phys. Rev. Lett. 93, 215703 (2004).

${ }^{10}$ A. Minoguchi, R. Richert, and C. A. Angell, J. Phys. Chem. B 108, 19825 (2005).

${ }^{11}$ C. A. Angell, Annu. Rev. Phys. Chem. 55, 559-583 (2004).

${ }^{12}$ C. A. Angell, Science 319, 582 (2008).

${ }^{13}$ P. Gallo, K. Amann-Winkel, C. A. Angell, M. A. Anisimov, F. Caupin, C. Chakravarty, E. Lascaris, T. Loerting, A. Z. Panagiotopoulos, J. Russo, J. A. Sellberg, H. E. Stanley, H. Tanaka, C. Vega, L. Xu, and L. G. M. Pettersson, Chem. Rev. 116, 7463 (2016).

${ }^{14}$ K. L. Ngai, S. Capaccioli, S. Ancherbak, and N. Shinyashiki, Philos. Mag. 91, 1809 (2011).

${ }^{15}$ D. R. MacFarlane and C. A. Angell, J. Phys. Chem. 88, 759 (1984).

${ }^{16}$ M. Nakanishi, P. Griffin, E. Mamontov, and A. P. Sokolov, J. Chem. Phys. 136, 124512 (2012).

${ }^{17}$ K. L. Ngai, J. Mol. Liq. 253, 113 (2018).

${ }^{18}$ K. L. Ngai, J. Chem. Phys. 109, 6982 (1998).

${ }^{19}$ K. L. Ngai and M. Paluch, J. Phys. Chem. 120, 857 (2004).

${ }^{20}$ S. Capaccioli, M. Paluch, D. Prevosto, K. L. Li-Min Wang, and J. Ngai, J. Phys. Chem. Lett. 3, 735 (2012).

${ }^{21}$ K. L. Ngai, Relaxation and Diffusion in Complex Systems (Springer, New York, 2011).

${ }^{22}$ N. Shinyashiki, S. Sudo, S. Yagihara, A. Spanoudaki, A. Kyritsis, and P. Pissis, J. Phys.: Condens. Matter 19, 205113 (2007).

${ }^{23}$ S. Capaccioli, K. L. Ngai, S. Ancherbak, P. A. Rolla, and N. Shinyashiki, J. NonCryst. Solids 357, 641-654 (2011).

${ }^{24}$ S. Capaccioli, K. L. Ngai, and N. Shinyashiki, J. Phys. Chem. B 111, 8197 (2007).

${ }^{25}$ K. L. Ngai, S. Capaccioli, and N. Shinyashiki, J. Phys. Chem. B 112, 3826 (2008).

${ }^{26}$ J. Swenson, H. Jansson, and R. Bergman, Phys. Rev. Lett. 96, 247802-247804 (2006).

${ }^{27}$ R. Bergman and J. Swenson, Nature 403, 283 (2000).

${ }^{28}$ H. Jansson, R. Bergman, and J. Swenson, J. Non-Cryst. Solids 351, 2858 (2005).

${ }^{29}$ S. Cerveny, G. A. Schwartz, R. Bergman, and J. Swenson, Phys. Rev. Lett. 93, 245702 (2004).

${ }^{30}$ S. Cerveny, F. Barroso-Bujans, A. Alegria, and J. Colmenero, J. Phys. Chem. C 114, 2604 (2010).

${ }^{31}$ J. Swenson and S. Cerveny, J. Phys.: Condens. Matter 27, 033102 (2015).

${ }^{32}$ S. Cerveny, F. Mallamace, J. Swenson, M. Vogel, and L. Xu, Chem. Rev. 116, 7608-7625 (2016).

${ }^{33}$ A. Panagopoulou, A. Kyritsis, N. Shinyashiki, and P. Pissis, J. Phys. Chem. B 116, 4593 (2012).

${ }^{34}$ A. Panagopoulou, A. Kyritsis, M. Vodina, and P. Pissis, Biochim. Biophys. Acta 1834, 977 (2013).

${ }^{35}$ P. Lunkenheimer, S. Emmert, R. Gulich, M. Köhler, M. Wolf, M. Schwab, and A. Loidl, Phys. Rev. E 96, 062607 (2017).

${ }^{36}$ A. Arbe, P. Malo de Molina, F. Alvarez, B. Frick, and J. Colmenero, Phys. Rev. Lett. 117, 185501 (2016).

${ }^{37}$ F. Parak, E. W. Knapp, and D. Kucheida, J. Mol. Biol. 161, 177 (1982).

${ }^{38}$ W. Doster, Biochim. Biophys. Acta 1804, 3-14 (2010).

${ }^{39}$ W. Doster, J. Non-Cryst. Solids 357, 622 (2011).

${ }^{40}$ E. Cornicchi, M. Marconi, G. Onori, and A. Paciaroni, Biophys. J. 91, 289-297 (2006).

${ }^{41}$ K. L. Ngai, S. Capaccioli, and A. Paciaroni, Chem. Phys. 424, 37-44 (2013).

${ }^{42}$ S. Capaccioli, K. L. Ngai, S. Ancherbak, and A. Paciaroni, J. Phys. Chem. B 116, 1745 (2012).

${ }^{43}$ K. L. Ngai, S. Capaccioli, and A. Paciaroni, J. Chem. Phys. 138, 235102 (2013).

${ }^{44}$ K. L. Ngai, S. Capaccioli, and A. Paciaroni, Biochim. Biophys. Acta 1861, 35533563 (2017).

${ }^{45}$ A. C. Drake, Y. Lee, E. M. Burgess, J. O. M. Karlsson, A. Eroglu, and A. Z. Higgins, PLoS One 13(1), e0190713 (2018). 
${ }^{46} \mathrm{M}$. Nakanishi and A. P. Sokolov, J. Non-Cryst. Solids 407, 478-485 (2015).

${ }^{47} \mathrm{~S}$. Ancherbak, "Dynamics of supercooled aqueous systems at low temperature and high pressure," Ph.D. dissertation (University of Pisa, 2011), https://etd.adm.unipi.it/t/etd-07022011-103037/.

${ }^{48}$ G. P. Johari, A. Hallbrucker, and E. Mayer, J. Chem. Phys. 97, 5851 (1992).

${ }^{49}$ G. P. Johari, J. Chem. Phys. 109, 1070 (1998).

${ }^{50} \mathrm{H}$. Jansson and J. Swenson, Eur. Phys. J. E 12, 51 (2003).

${ }^{51}$ J. Swenson, H. Jansson, W. S. Howells, and S. Longeville, J. Chem. Phys. 122, 084505 (2005).

${ }^{52}$ J. Hedström, J. Swenson, R. Bergman, H. Jansson, and S. Kittaka, Eur. Phys. J.: Spec. Top. 141, 53-56 (2007).

${ }^{53}$ J. Banys, M. Kinka, A. Meskauskas, J. Macutkevic, G. Völkel, W. Böhlman, V. Umamamaheswari , M. Hartmann, and A. Pöppl, Ferroelectrics, 318, 201-207 (2005).

${ }^{54}$ S. Cerveny, S. Arrese-Igor, J. S. Dolado, J. J. Gaitero, A. Alegria, and J. Colmenero, J. Chem. Phys. 134, 034509 (2011).

${ }^{55}$ M. Cammarata, M. Levantino, A. Cupane, A. Longo, A. Martorana, and F. Bruni, Eur. Phys. J. E 12, 63 (2003).

${ }^{56}$ M. Oguni, Y. Kanke, and S. Namba, AIP Conf. Proc. 982, 34 (2008).

${ }^{57}$ M. Oguni, S. Maruyama, K. Wakabayashi, and A. Nagoe, Chem.-Asian J. 2, 514 (2007).

${ }^{58}$ M. Sun, L.-M. Wang, Y. Tian, R. Liu, K. L. Ngai, and C. Tan, J. Phys. Chem. B 115, 8242 (2011).

${ }^{59}$ J. B. Hasted, "Liquid water: Dielectric properties," in Water: A Comprehensive Treatise, edited by F. Franks (Plenum, New York, 1972).

${ }^{60}$ R. Buchner, J. Barthel, and J. Stauber, Chem. Phys. Lett. 306, 57 (1999).

${ }^{61}$ C. Rønne, P. O. Åstrand, and S. R. Keidung, J. Chem. Phys. 107, 5319 (1997).

${ }^{62}$ D. Bertolini, M. Cassettari, and G. Salvetti, J. Chem. Phys. 76, 3285 (1982).

${ }^{63}$ N. Shinyashiki, M. Shimomura, T. Ushiyama, T. Miyagawa, and S. Yagihara, J. Phys. Chem. B 111, 10079 (2007).

${ }^{64}$ S. Sudo, M. Shimomura, T. Saito, T. Kashiwagi, N. Shinyashiki, and S. Yagihara, J. Non-Cryst. Solids 305, 197 (2002).

${ }^{65}$ S. Sudo, M. Shimomura, K. Kanari, N. Shinyashiki, and S. Yagihara, J. Chem. Phys. 124, 044901 (2006).

${ }^{66}$ S. Sudo, S. Tsubotani, M. Shimomura, N. Shinyashiki, and S. Yagihara, J. Chem. Phys. 121, 7332 (2004).

${ }^{67}$ N. Shinyashiki, M. Shinohara, Y. Iwata, T. Goto, M. Oyama, S. Suzuki, W. Yamamoto, S. Yagihara, T. Inoue, S. Oyaizu, S. Yamamoto, K. L. Ngai, and S. Capaccioli, J. Phys. Chem. B 112, 15470 (2008).

${ }^{68} \mathrm{~K}$. Grzybowska, M. Paluch, A. Grzybowski, S. Pawlus, S. Ancherbak, D. Prevosto, and S. Capaccioli, J. Phys. Chem. Lett. 1, 1170 (2010).

${ }^{69}$ S. Cerveny, J. Colmenero, and A. Alegría, Macromolecules 38, 7056 (2005).
${ }^{70}$ S. K. Jain and G. P. Johari, J. Phys. Chem. 92, 5851 (1988).

${ }^{71}$ S. Cerveny, A. Alegría, and J. Colmenero, J. Chem. Phys. 128, 044901 (2008). ${ }^{72}$ J. Sjöström, J. Mattsson, R. Bergman, E. Johansson, K. Josefsson, D. Svantesson, and J. Swenson, Phys. Chem. Chem. Phys. 12, 10452 (2010).

${ }^{73}$ K. Pathmanathan and G. P. Johari, J. Polym. Sci., Part B: Polym. Phys. 28, 675 (1990).

${ }^{74}$ K. Pathmanathan and G. P. Johari, J. Chem. Soc. Faraday Trans. 90, 1143 (1994).

${ }^{75}$ S. Cerveny, I. Combarro-Palacios, and J. Swenson, J. Phys. Chem. Lett. 7, 40934098 (2016).

${ }^{76}$ N. Shinyashiki, W. Yamamoto, A. Yokoyama, T. Yoshinari, S. Yagihara, R. Kita, K. L. Ngai, and S. Capaccioli, J. Phys. Chem. B 113, 14448 (2009).

${ }^{77}$ S. Khodadadi, S. Pawlus, J. H. Roh, V. Garcia Sakai, E. Mamontov, and A. P. Sokolov, J. Chem. Phys. 128, 195106 (2008).

${ }^{78} \mathrm{H}$. Jansson and J. Swenson, Biochim. Biophys. Acta 1804, 20 (2010).

${ }^{79}$ S. A. Lusceac, M. R. Vogel, and C. R. Herbers, Biochim. Biophys. Acta 1804, 41-48 (2010).

${ }^{80}$ F. G. Parak and G. U. Nienhaus, J. Non-Cryst. Solids 131-133, 362-368 (1991).

${ }^{81}$ W. Doster, Eur. Biophys. J. 37, 591-602 (2008).

${ }^{82}$ A. Panagopoulou, A. Kyritsis, R. Sabater i Serra, J. L. Gomez, Ribellez, N. Shinyashiki, and P. Pissis, Biochim. Biophys. Acta 1814, 1984-1996 (2011).

${ }^{83}$ S. Khodadadi and A. P. Sokolov, Soft Matter 11, 4984 (2015).

${ }^{84}$ H. Jansson, R. Bergman, and J. Swenson, J. Phys. Chem. B 109, 24134 (2005).

${ }^{85}$ C. Gainaru, A. Fillmer, and R. Böhmer, J. Phys. Chem. B 113, 12628 (2009).

${ }^{86}$ I. Combarro Palacios, C. Olsson, C. S. Kamma-Lorger, J. Swenson, and S. Cerveny, J. Chem. Phys. 150, 124902 (2019).

${ }^{87}$ W. Doster, H. Nakagawa, and M. S. Appavou, J. Chem. Phys. 139, 045105 (2013).

${ }^{88}$ W. Doster and M. Settles, Biochim. Biophys. Act. 1749, 173-186 (2005).

${ }^{89}$ H. Lichtengegger, W. Doster, T. Kleinert, A. Birk, B. Sepiol, and G. Vogl, Biophys. J. 76, 414-422 (1999).

${ }^{90}$ S. Khodadadi, S. Pawlus, and A. P. Sokolov, J. Phys. Chem. B 112, 14273-14280 (2008).

${ }^{91}$ S. Khodadadi, A. Malkovskiy, A. Kisliuk, and A. P. Sokolov, Biochim. Biophys. Acta 1804, 15-19 (2010).

${ }^{92}$ M. Wubbenhorst and J. van Turnhout, J. Non-Cryst. Solids 305, 40-49 (2002).

${ }^{93}$ K. Wood, C. Caronna, P. Fouquet, W. Haussler, F. Natali, J. Ollivier, A. Orecchini, M. Plazanet, and G. Zaccai, Chem. Phys. 345, 305 (2008).

${ }^{94}$ R. F. Tilton, J. C. Dewan, and G. A. Petsko, Biochemistry 31(9), 2469-2481 (1992).

${ }^{95}$ R. Bohmer, K. L. Ngai, C. A. Angell, and D. J. Plazek, J. Chem. Phys. 99, 4201 (1993).

${ }^{96}$ D. Ringe and G. A. Petsko, Biophys. Chem. 105, 667-680 (2003). 